\title{
TLR3 preconditioning enhances the
} therapeutic efficacy of umbilical cord mesenchymal stem cells in TNBS-induced colitis via the TLR3-Jagged-1-Notch-1 pathway

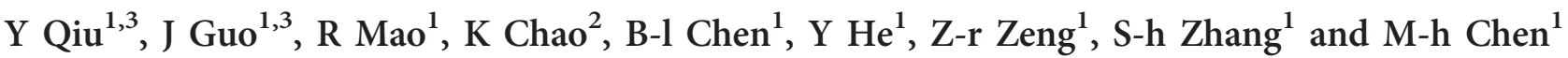

Toll-like receptor-3 (TLR3) priming may enhance mesenchymal stem cell (MSC) immunosuppressive activity, but this mechanism has not been investigated in the context of inflammatory bowel disease. Thus, we assessed the immunosuppressive properties of TLR3-primed MSCs using a trinitrobenzene sulfonate (TNBS)-induced mouse model of colitis. Intraperitoneally injected polyribocytidylic acid (poly (I:C)- (a ligand of TLR3) primed human umbilical cord-derived MSCs (hUC-MSCs) migrated to the inflamed colon and effectively improved clinical and pathological manifestations in colitic mice compared with mice treated with unstimulated hUC-MSCs (UCMs). Poly (I:C)-MSCs decreased a wide range of inflammatory cytokines and increased systemic interleukin-10 (IL-10) levels in colonic tissues. Poly (I:C)-MSCs also impaired T-helper type 1/17 (Th1/17) cell expansion and enhanced the suppressive effects of regulatory Tcells (Treg) in vitro and in vivo. Poly (I:C)-MSCs suppressed the proliferation of activated mesenteric lymph node (MLN) cells via the overproduction of prostaglandin $E_{2}\left(P E_{2}\right)$ and upregulation of Jagged-1. $P G E_{2}$ produced by hUC-MSCs in response to poly $(I: C)$ increased the production of IL-10 and promoted the differentiation of Treg, which could be reversed by inhibition of Notch-1. Collectively, preconditioning MSCs with poly (I:C) enhanced the therapeutic effects of hUC-MSCs in TNBS-induced colitis, and TLR3-activated Notch-1 signaling regulated the immune suppression of hUC-MSCs through the production of $\mathrm{PGE}_{2}$.

\section{INTRODUCTION}

Inflammatory bowel disease (IBD) is characterized by the overproduction of inflammatory cytokines by immune cells, which propagates inflammation and tissue damage in the gut. ${ }^{1}$ Increased knowledge regarding the etiopathogenesis of IBD has led to the emergence of novel therapeutic strategies. ${ }^{2}$ Among these approaches, the use of human mesenchymal stem cells (hMSCs) represents a unique, cell-based strategy to induce anti-inflammatory effects in the gut with the potential of maintaining intestinal homeostasis. ${ }^{3}$ However, the effects of MSCs on immune-related disorders have been challenged in several in vivo studies in murine models. ${ }^{4,5}$ This partly results in the function and survival of MSCs being impaired by inflamed microenvironments, thereby limiting their therapeutic potential. $^{6-8}$ Taking advantage of the immunosuppressive rather than the immunostimulating properties of MSCs depends on a complex balance among diverse stimuli, which may functionally polarize MSCs, although such properties are essential for using MSCs in clinical practice. ${ }^{9}$ Another significant determinant of the therapeutic potential of MSCs is the insufficient trapping of MSCs in injured tissues after systemic administration. Both of these attributes, immune modulation and migration of MSCs, are markedly affected by specific toll-like receptors (TLRs). ${ }^{10}$

Some evidence points to TLR3 as primarily mediating the stress migration responses of hMSCs. TLR3 is highly expressed

${ }^{1}$ Department of Gastroenterology, The First Affiliated Hospital of Sun Yat-Sen University, Guangzhou, People's Republic of China and ${ }^{2}$ Department of Gastroenterology, The Sixth Affiliated Hospital of Sun Yat-Sen University, Guangzhou, People's Republic of China. Correspondence: S-h Zhang (shenghongzhang@163.com) or M-h Chen (chenminhu@vip.163.com)

${ }^{3}$ The first two authors contributed equally to this work.

Received 18 November 2015; accepted 19 July 2016; published online 21 September 2016. doi:10.1038/mi.2016.78 
in this cell population, ${ }^{11}$ and migration assays have demonstrated that exposing hMSCs to the TLR3 ligand or poly (I:C) enhances their migration compared with other TLR ligands. ${ }^{12}$ Moreover, the activation of TLR3 was found to amplify MSC trophic factors and enhance their therapeutic potency in murine models of myocardial damage and diabetic neuropathy. ${ }^{13,14}$ The TLR triggering effects appear to be related to the MSC signaling of Notch receptors in T cells. Indeed, MSCs express the Notch ligand Jagged-1, and Jagged-1 mediates the inhibition of nuclear factor- $\mathrm{KB}$ signaling, ${ }^{15}$ thereby inhibiting Notch signaling from hampering the suppressive activity of MSCs on T cells. ${ }^{16}$ Furthermore, Notch signaling regulates CXCR4 expression and the migration of MSCs. ${ }^{17}$ However, whether TLR3 priming of MSCs may be instrumental in enhancing the immunosuppressive effects of MSCs in the context of IBD is currently unknown.

Therefore, we examined the effect of TLR pretreatment of MSCs in vivo using a mouse model of colitis. We found that short in vitro TLR3-preconditioning enhances the protective effect of human umbilical cord-derived MSCs (hUC-MSCs) against trinitrobenzene sulfonic acid (TNBS)-induced colitis in mice by producing prostaglandin $\mathrm{E}_{2}\left(\mathrm{PGE}_{2}\right)$ via the TLR3Jagged-1-Notch-1 pathway.

\section{RESULTS \\ Poly (I:C) enhanced the protective effect of hUC-MSCs against TNBS-induced colitis}

Intraperitoneal injection of hUC-MSCs prevented the loss of body weight and decreased the mortality of mice compared with phosphate-buffered saline control injection (Figure 1a,b). Treatment with poly (I:C)-stimulated hUC-MSCs (poly (I:C)MSCs) restored $90 \%$ of the body weight and rescued $100 \%$ of the lethality of TNBS-induced colitic mice, which were significantly higher compared with mice treated with hUCMSCs without poly (I:C) preconditioning (Figure 1a,b).

In line with these observations, histopathology of the colons of TNBS-treated mice killed on day 4 exhibited a reduction in colon length compared with the ethanol-treated mice, which was modestly restored by treatment with hUC-MSCs. However, at the two time-points examined, there was an additional significant improvement in colon length owing to treatment with poly (I:C)-MSCs compared to hUC-MSCs (Figure 1c,d).
Moreover, the colitis clinical score was significantly diminished in the poly (I:C)-MSC-treated mice compared with the mice treated with UCMs (Figure 1e).

Histologic examination of the colons showed that hUC-MSC treatment reduced TNBS-induced transmural inflammation, mucin-producing goblet and epithelial cells, the focal loss of crypts (Figure 1f,g), infiltration of neutrophil (myeloperoxidase (MPO) activity; Figure 1h), and inflammatory $\mathrm{CD} 4{ }^{+}$lymphocytes in the lamina propria Figure 1i). All these attributes were significantly more inhibited by poly (I:C)-MSC treatment compared with treatment with non-preconditioned MSCs (Figures 1f-i).

In contrast, hUC-MSCs pretreated with lipopolysaccharide (LPS, a ligand for TLR4) or tumor necrosis factor- $\alpha$ (TNF- $\alpha$ ) (to mimic the inflammatory environment) did not decrease lethality or body weight loss in the colitic mice, nor did it ameliorate the reduction in colon length or the histologic damage observed upon treatment with poly (I:C)-MSCs (Figures 1a-i). IFN- $\gamma$ priming also induced enhanced protective effects, which was consistent with a previous report. ${ }^{5}$ We next examined the efficiency of hUC-MSCs during the later phases of the disease when colitis is fully established. hUC-MSCs and poly (I:C)-MSC injection after disease onset partially reversed disease evolution (Figure 1j).

\section{Poly (I:C) enhanced the anti-inflammatory activity of hUC- MSCs in the colon of colitic mice by shifting the Treg/Th1/ Th17 profile}

We next investigated the cytokine profile in the TNBS colitic mice following MSC injections. Treatment with hUC-MSCs reduced the production of Th1/17 signature cytokines, such as interferon- $\gamma$ (IFN- $\gamma)$, IL-17A, IL-21, and IL-23, in the colons of TNBS-treated mice, which was accompanied by downregulation of the systemic inflammatory response (Figure 2a,b). Poly (I:C) preconditioning enhanced the ability of hUC-MSCs to suppress these Th1/17 signature cytokines, whereas priming with other ligands did not induce a similar effect on the production of inflammatory mediators (Figure 2a,b). In addition, hUC-MSC treatment significantly increased IL-10 production in the colon, and this increase in IL-10 production was more significant when treated with poly (I:C)-stimulated hUC-MSC (Figure 2a,b).

\footnotetext{
Figure 1 Short in vitro poly (I:C)-primed MSCs, but not Pam $\mathrm{CSK}_{4}$, LPS, or TNF- $\alpha$, enhanced the protective effect of hUC-MSCs against TNBS-induced colitis. Colitic BALB/c mice were treated with xenogenic (from human umbilical cord) hUC-MSCs or syngeneic (from adipose of BALB/c mice) mASCs ( $10^{6}$ cells per mouse) $2 \mathrm{~h}$ after rectal administration of TNBS. As a control, mice were injected with hUC-MSCs pretreated with poly (I:C), TNF- $\alpha$, LPS, or IFN- $\gamma 2 \mathrm{~h}$ after rectal administration of TNBS, as designated in (a-i). (a-e) The clinical evolution and severity of colitis were monitored for 7 days. (a) Mantel-Cox analysis of survival rate; (b) percentage of body weight loss; (c and d) colon length measurements on days 4 and 7; (e) colitis score for colitis severity. (f and $\mathbf{g}$ ) Histopathologic analysis of the colons on day 4. Histology scores were derived from microscopic analyses of longitudinal colon sections from each mouse. (h) Neutrophil infiltration was determined via a colonic MPO activity assay. (i) Inflammatory T-lymphocyte infiltration was measured by counting the number of cells per microscopic field on immunostained colon sections. (j) hUC-MSC treatment abrogates established colitis. Colitis induced with TNBS ( 3 mg per mouse) at day 0 was treated from day 4 (arrow) with hUC-MSCs $\left(10^{6}\right.$ mouse per day). ${ }^{*} P<0.05 ;{ }^{* *} P<0.01$. Five mice per group were used. The results are shown as the mean \pm s.d. Mice treated only with ethanol were served as control of TNBS-induced colitis. mASCs isolated from adipose of BALB/c mice were used as a syngeneic control to xenogenic hUC-MSC isolated from human umbilical cord. BALB/c, Bagg albino/c mice; $\mathrm{EtOH}$, ethanol; hUC-MSCs, human umbilical cord-derived MSCs; IFN- $\gamma$, interferon- $\gamma$; LPS, lipopolysaccharide, a ligand for TLR4; mASC, mice adipose stem cell; MLN, mesenteric lymph node; MNC, mononuclear cell; MPO, myeloperoxidase; MSC, mesenchymal stem cell; poly (I:C)-MSCs, polyribocytidylic acid-stimulated hUC-MSCs; TNBS, trinitrobenzene sulfonic acid; TNF- $\alpha$, tumor necrosis factor- $\alpha$.
} 
a
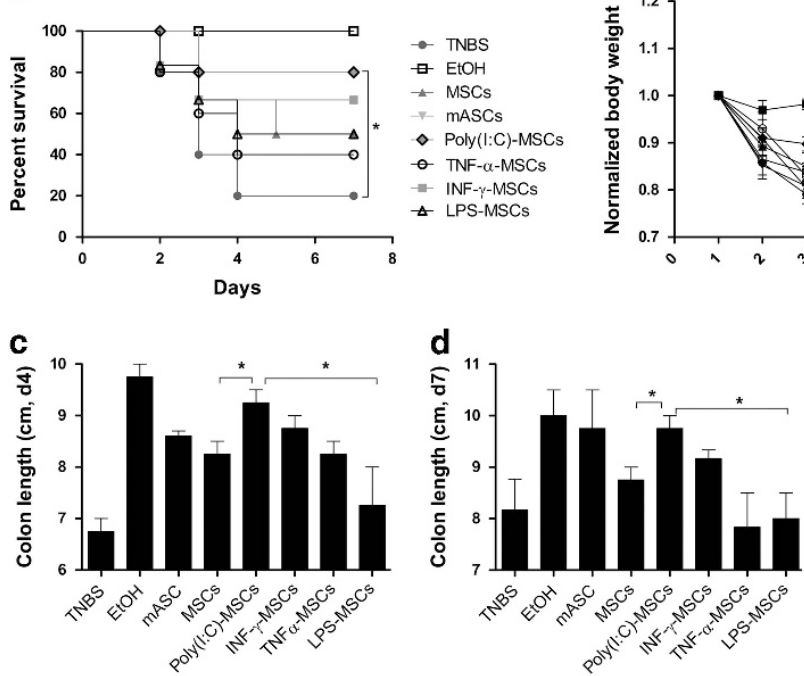
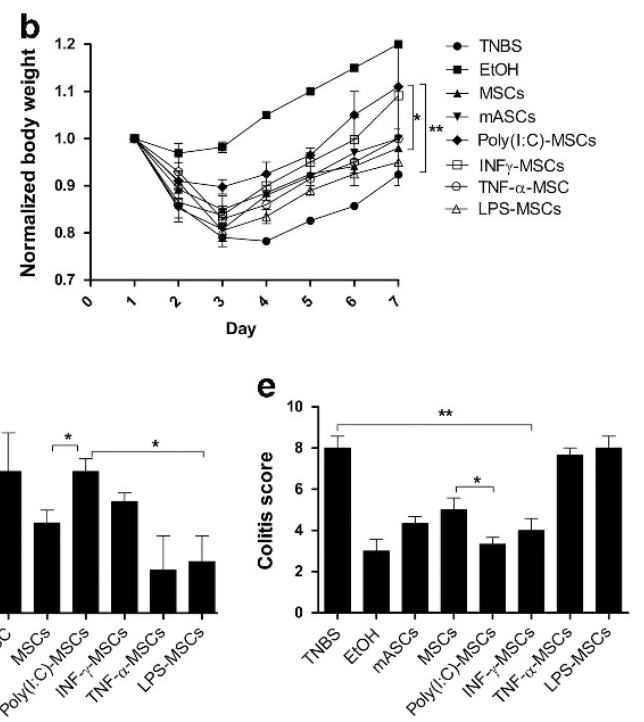

f

EtOH

TNBS

Poly(I:C)-MSCs

LPS-MSCs

TNF- $\alpha-M S C s$
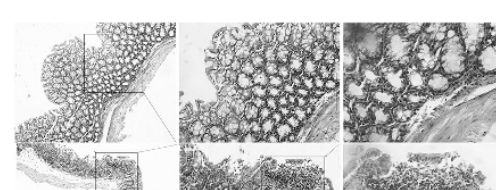

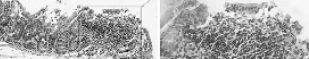
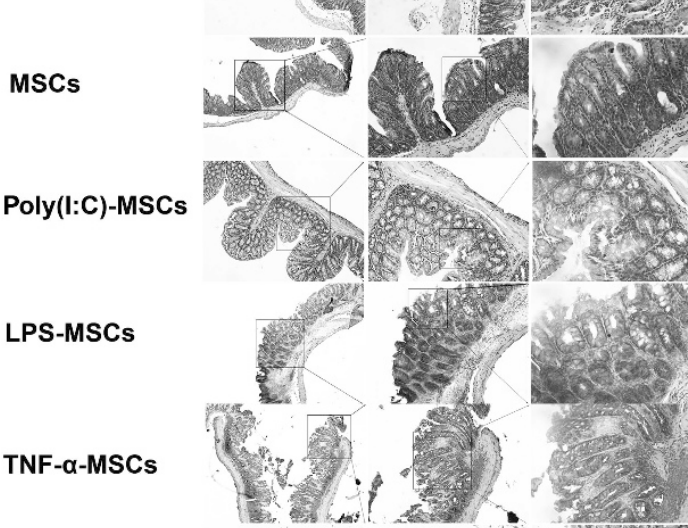

INF-Y-MSCs

mASCs

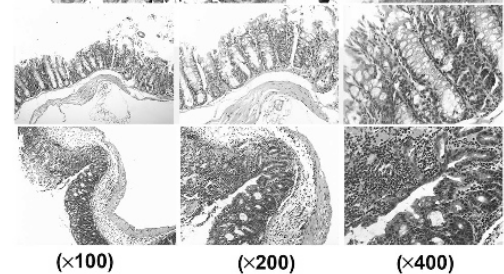

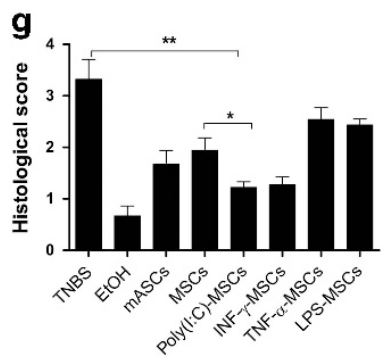
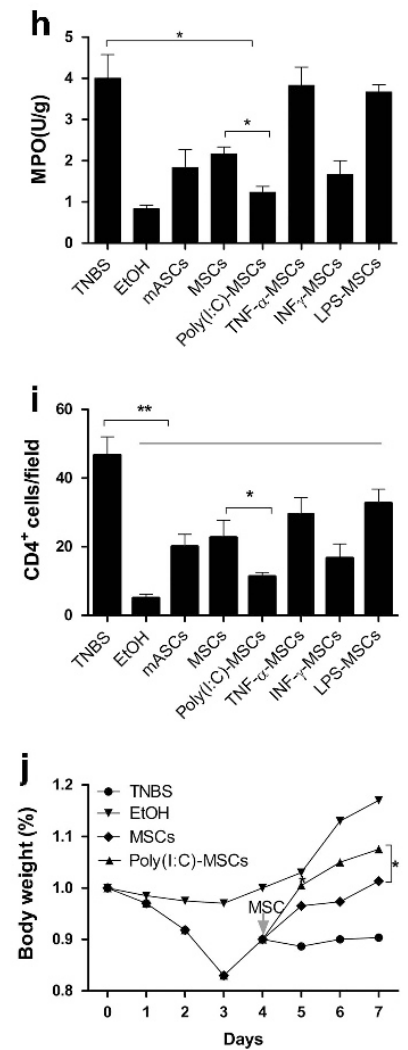

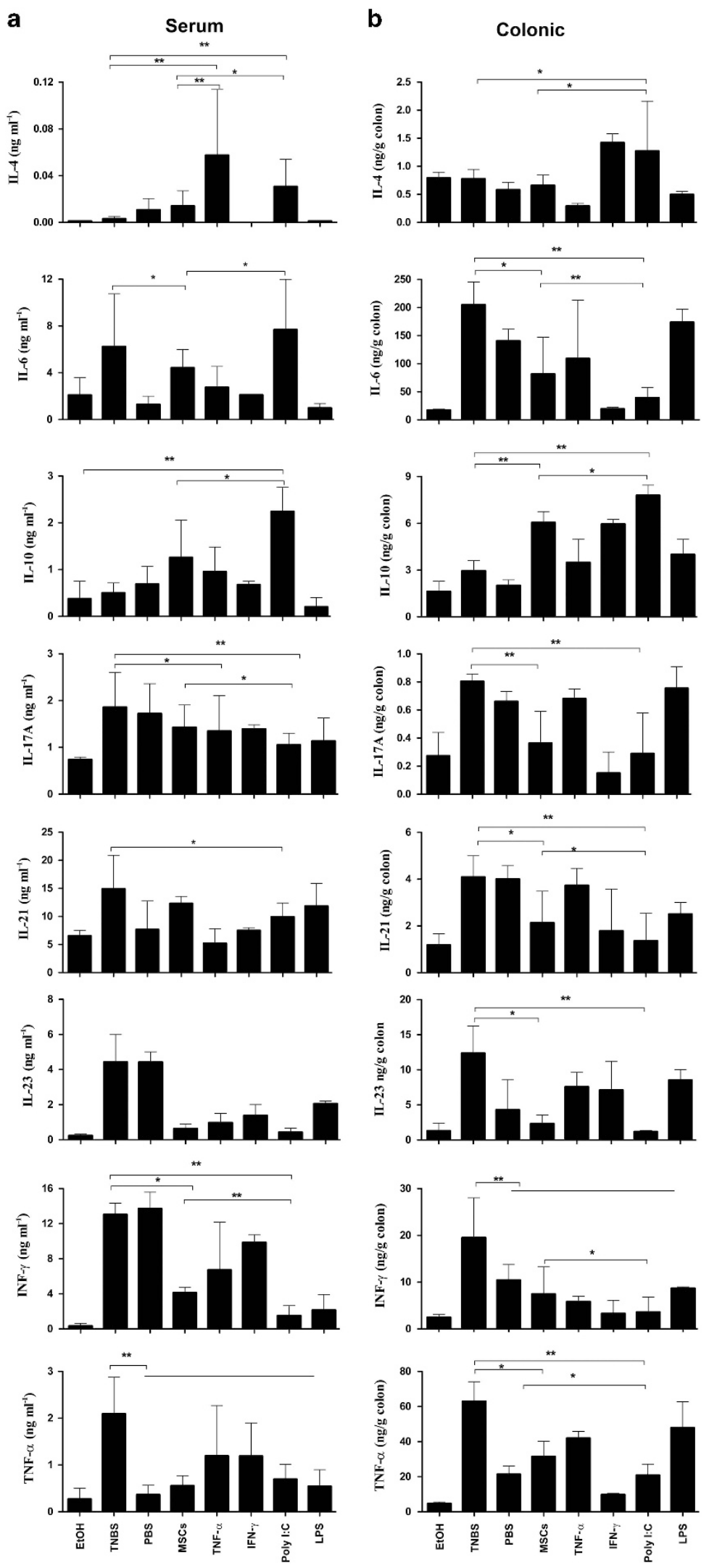
We next investigated whether the decrease in the inflammatory mediators was only a consequence of the diminished inflammatory infiltration in the colonic mucosa of the poly (I:C)-MSC-treated mice (Figure 1h,i). To determine whether hUC-MSCs affected CD4 ${ }^{+}$T-cell polarization in the colons of the mice, the colonic infiltration of Th1/2/17 and Treg (defined as $\mathrm{CD}^{+}{ }^{+} \mathrm{CD} 25^{+}$fork-head box p3-positive (Foxp3 ${ }^{+}$) cells) was examined via flow cytometry. The administration of hUCMSCs led to increased localization of Treg in the colon along with decreased infiltration of pathogenic Th1/17 subsets (Figure 2c,d).

Poly (I:C)-MSC-treated colitic mice had significantly higher amounts of Treg in their MLNs compared with TNBS mice and MSC-treated mice (Figure 2c). Moreover, poly (I:C)-MSCs
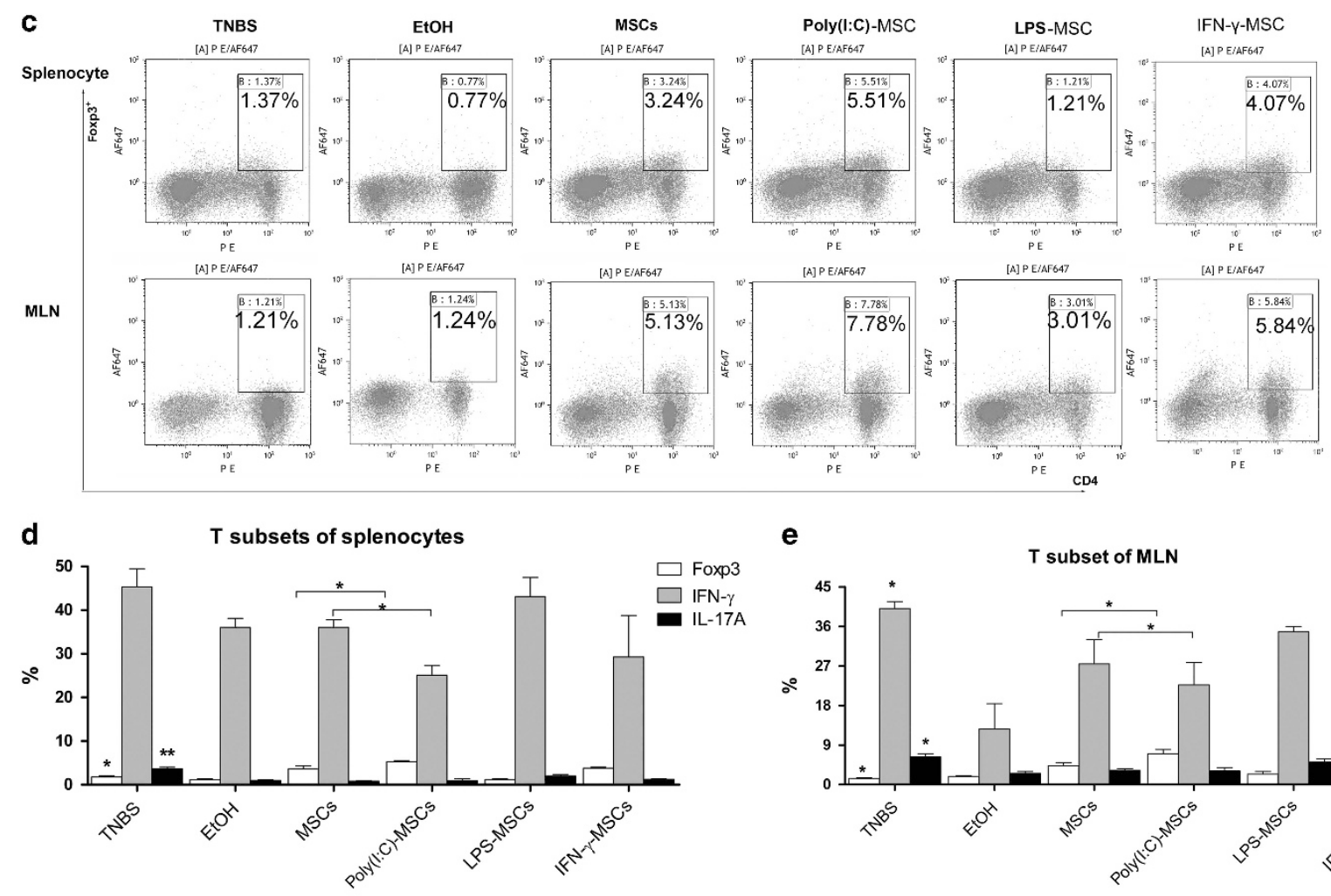

$\mathbf{e}$
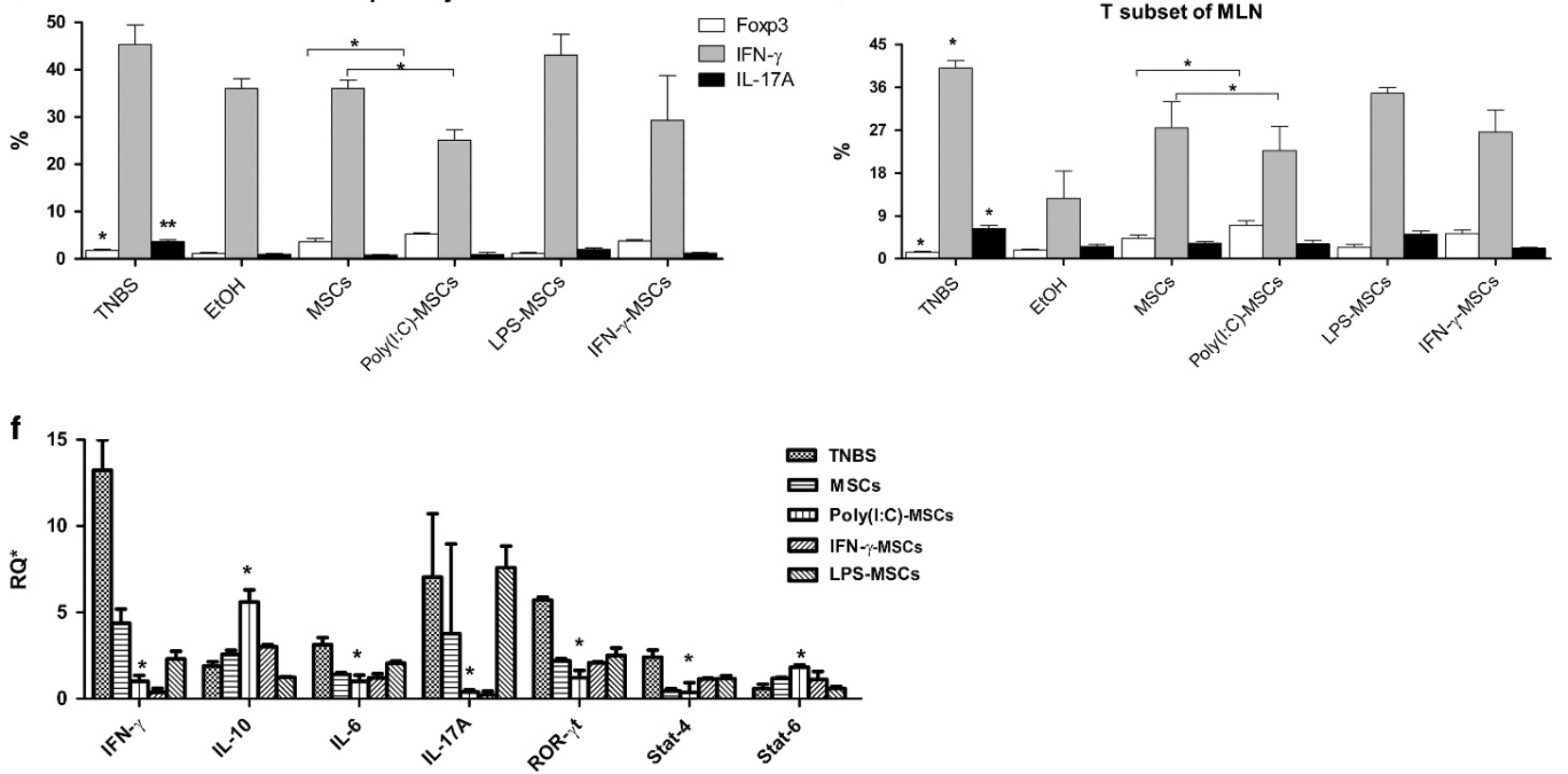

Figure 2 (Continued).

Figure 2 Poly (I:C)-MSCs downregulated inflammatory responses by shifting the Treg/Th17 profile in experimental colitis. (a and b) TNBS-induced colitic mice were injected intraperitoneally with hUC-MSCs or with hUC-MSC pretreated with poly (I:C), TNF- $\alpha$, LPS, or IFN- $\gamma$. Protein extracts were obtained from the (a) serum and (b) colons on day 4, and cytokine levels were measured using customized Luminex-based multiplex immunoassay kits. (c-e) MLN and splenocytes isolated on day 4 were analyzed for expressions of Foxp3, Th1, T17, and Treg (staining for Foxp3 ${ }^{+} /$IFN- $\gamma /$ IL-17 in gated CD4 $\mathrm{T}^{+}$cells) via flow cytometry. (f) Changes in the mRNA expressions of Th1/2/17 and Treg-related inflammatory mediators following the various treatments. Acute TNBS colitis demonstrated a cytotoxic and chemotactic profile with significantly elevated mRNA expressions of IL-17, IFN- $\gamma$, IL-6, ROR $\gamma$ t, and Stat- 6 compared with controls. Preventive treatment with hUC-MSCs or poly(I:C)-MSCs reduced the levels of the above cytokines. The data are presented as the means \pm s.d. of three separate experiments in which five mice per group were analyzed $\left({ }^{\star} P<0.05 ;{ }^{* \star} P<0.01\right)$. EtOH, ethanol; hUCMSC, human umbilical cord-derived MSC; IFN, interferon; LPS, lipopolysaccharide; mASC, mice adipose stem cell; MLN, mesenteric lymph node; MNC, mononuclear cell; MSC, mesenchymal stem cell; naïve, unstimulated hUC-MSC; poly (I:C)-MSC, polyribocytidylic acid-stimulated hUC-MSC; ROR $\gamma$, retinoic acid receptor-related orphan receptor $\gamma$ t; Stat-5, signal transducer and activator of transcription 4; Th17, T-helper type 17; TNBS, trinitrobenzene sulfonic acid; TNF- $\alpha$, tumor necrosis factor- $\alpha$; Treg, regulatory $\mathrm{T}$ cell. 


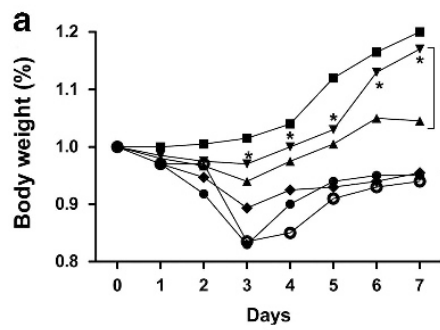

$-\mathrm{TNBS}$
$-\mathrm{EtOH}$

$\star$ MSCs

- Poly (I:C)-MSCs

- siRNA-MSCs

- DAPT-MSCs ${ }^{+}$poly $(\mathrm{I}: \mathrm{C})$
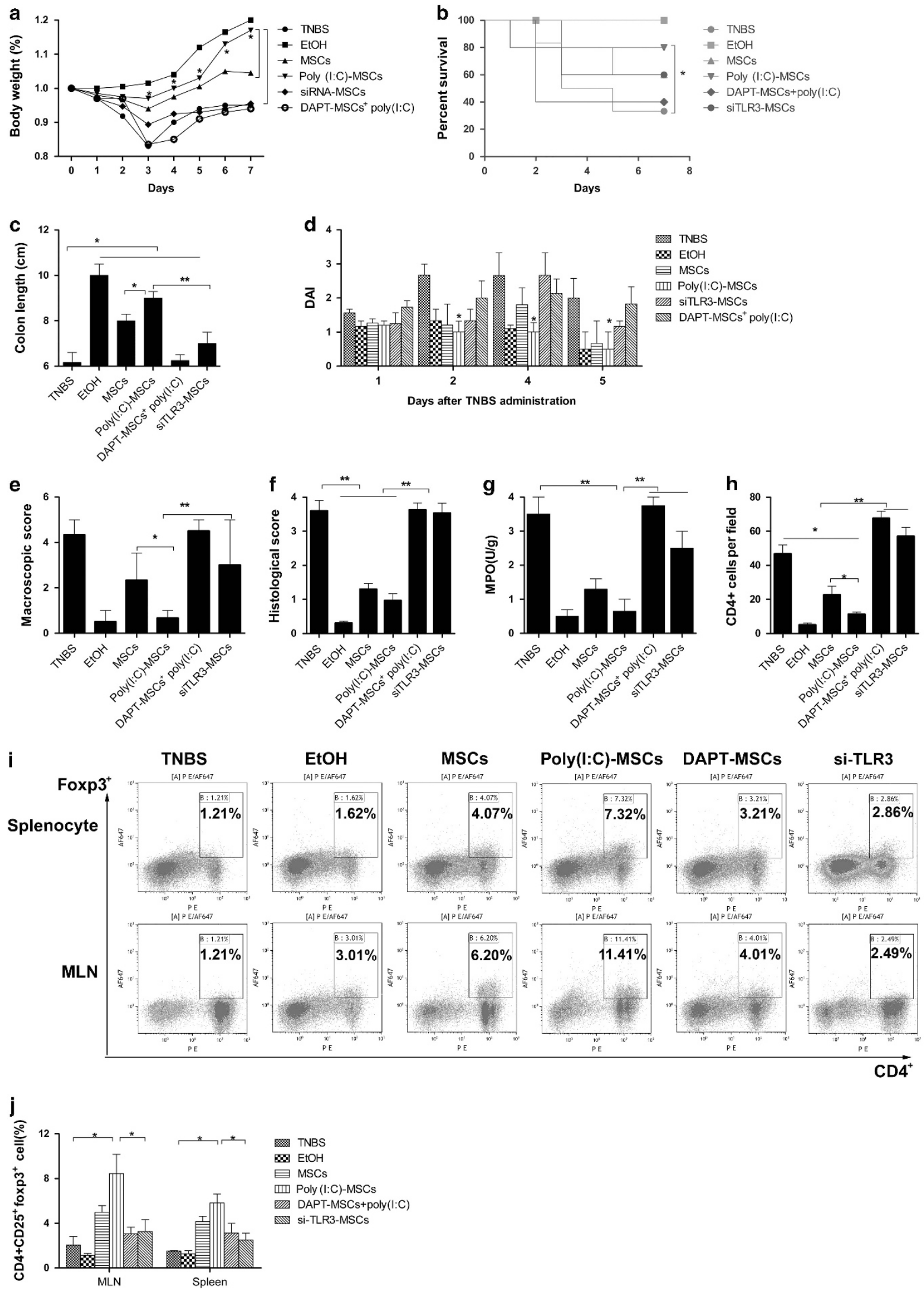
diminished the number of autoreactive IFN- $\gamma$ - and IL-17Asecreting $\mathrm{T}$ cells and increased the number of regulatory IL-10producing Treg cells (Figure 2d). Acute TNBS colitis was characterized by a cytotoxic profile with significantly elevated mRNA expressions of IFN- $\gamma$, IL-17A, IL-6, retinoic acid receptor-related orphan receptor $\gamma \mathrm{t}(\mathrm{ROR} \gamma \mathrm{t})$, and signal transducer and activator of transcription 4 (Stat-4) compared with controls (Figure 2f). Reductions in Th1 and Th17 activities after poly (I:C)-MSC transplantation were further confirmed by the expressions of Stat-4, IL-6, IL-17A, and ROR $\gamma \mathrm{t}$ (Figure 2f). A possible role of poly(I:C)-MSCs in promoting the Th2 subset was confirmed by analyzing the Th2 lineage transcription factor Stat- $6 .{ }^{18}$ Collectively, these results indicated that poly (I:C)-MSCs downregulated inflammatory responses by shifting the Treg/Th1/Th17 profile in an experimental model of colitis.

\section{TLR3 and Notch-1 had important roles in the function of hUC-MSCs in TNBS-induced colitis}

We next sought to further substantiate the role of TLR3 in the priming effect of MSCs. When TLR3 was downregulated by small interfering RNA (siRNA) or by $\mathrm{NN}$-(3,5-difluorophenacetyl-L-alanyl)- $S$-phenylglycine $t$-butyl ester (DAPT), an inhibitor of Notch signaling, poly (I:C)-MSCs no longer improved the decreases in body weight, survival rate, the disease activity index, and the colitis score in experimental colitic mice (Figure 3a-e).

As expected, the administration of TLR3 siRNA- or DAPTtreated hUC-MSCs neither prevented histologic damage (Figure 3f) nor decreased neutrophil (Figure 3g) or inflammatory T-lymphocyte infiltration (Figure $3 \mathbf{h}$ ). In addition, it also did not increase the colonic infiltration of Treg cells, as opposed to when TLR3 and Notch signaling were not inhibited (Figure 3i,j).

\section{Poly (I:C) enhances the migration of hUC-MSCs to inflammatory sites}

To ascertain the migration of hUC-MSC to the inflamed colon, the colonic presence of intraperitoneal injected luciferaseexpressing fluc-hUC-MSCs was investigated. Fluc-hUC-MSCs were detected in inflamed colons but not in noninflamed colons (Figure 4a).

To better understand the half-life and trafficking of the infused hUC-MSCs, we injected CM-DiI-labeled hUC-MSCs and detected the inoculated cells in the MLNs and spleens of the recipient mice 1-7 days after injection (Figure 4b). Importantly, poly (I:C) stimulation enhanced the trafficking of hUC-MSCs to the inflamed colon, which was suppressed by siRNA transfection targeting TLR3 or DAPT targeting Notch-1 (Figure $4 \mathbf{b}$ ).

To better explore the in vivo distribution of hUC-MSCs, we next detected infused 5, 6-carboxy fluorescein succinimidyl ester (CFSE)-labeled MSC cells in various organs by flow cytometry. This experiment showed that poly (I:C) stimulation enhanced the trafficking of hUC-MSCs to inflamed colons but not to the spleen, duodenum, or MLN (Figure 4c). In addition, we found that the preferential homing of poly (I:C)-MSCs to inflamed tissues correlated with the expression of CCR10 (Figure 4d). In addition to enhancing MSC trafficking in vivo, the activation of TLR3 signaling also promoted the migration of MSCs in transwell plates in vitro (Figure 4e).

\section{Poly (I:C) enhanced the inhibitory activity of hUC-MSCs against the proliferation of MNCs}

To gain further insight regarding the mechanism of MSC preconditioning to enhance their immunosuppressive effects, we next investigated MSC inhibition of MNC proliferation. Presimulation with TLR2/4 agonists $\left(\mathrm{Pam}_{3} \mathrm{CSK}_{4}\right.$ and LPS) did not alter the inhibitory effect of hUC-MSCs on MNC proliferation, whereas priming with poly (I:C) significantly increased MNC inhibition of MNC proliferation (Figure 5a,b). In addition, this proliferation arrest was abolished by siRNA against TLR3 (Figure 5c).

Because soluble factors can also mediate the immunosuppressive activity of MSCs, we next examined whether soluble factors produced by hUC-MSCs could influence MNC proliferation. The proliferation of MNCs was slightly inhibited in the presence of supernatant from culture media from UCMs, but was further suppressed in the presence of culture media obtained from hUC-MSCs stimulated with poly (I:C) (poly (I:C)-CM) (Figure 5d). Again, inhibited proliferation was reversed by inhibition of TLR3 (Figure 5e).

These findings indicate that soluble factors selectively induced by TLR3 priming participate in conferring the immunosuppressive effect of hUC-MSCs.

\section{$\mathrm{PGE}_{2}$ produced by hUC-MSCs in response to poly $(\mathrm{I}: \mathrm{C})$ was responsible for MNC suppression}

To explore the soluble factors excreted by hUC-MSCs that inhibit the proliferation of MNCs, an enzyme-linked immunosorbent assay (ELISA) analysis was performed. The results

\footnotetext{
Figure 3 Poly (I:C) enhanced the protective effect of hUC-MSCs against TNBS-induced colitis, which was abolished by TLR3 deficiency or Notch-1 inhibition. (a) Survival rates were compared among the groups of treated mice. (b) Body weight changes were calculated as a percentage of initial body weight on day 0. (c) Colon lengths were measured on day 4; (d) DAI for colitis severity; (e) macroscopic score for colitis severity on day 4; (f) histopathologic analysis of the colons on day 4 . Histology scores were derived from microscopic analyses of longitudinal colon sections from each mouse. (g) Neutrophil infiltration was determined by colonic MPO activity assay on day 4. One unit of MPO activity represents the amount of enzyme that reduces $1 \mu \mathrm{mol}$ peroxide/min. (h) Inflammatory T lymphocyte infiltration was measured by counting the number of cells per microscopic field on immunostained colon sections. (i and j) Expression of CD4 ${ }^{+} \mathrm{CD} 25^{+}$Foxp ${ }^{+}$in cells was determined via flow cytometry on day $4 .{ }^{\star} P<0.05$; ${ }^{* *} P<0.01$. Results are shown as the means \pm s.d. DAl, disease activity index; DAPT, $N N$-(3,5-difluorophenacetyl-L-alanyl)-S-phenylglycine $t$-butyl ester, a $\gamma$-secretase inhibitor that inhibits Notch signaling; hUC-MSC, human umbilical cord-derived MSC; MPO, myeloperoxidase; poly (I:C)-MSC, polyribocytidylic acid-stimulated hUCMSC; siTLR3, TLR3 signaling in MSCs was inhibited by downregulating TLR3 expression with small RNA interference (siRNA); TLR3, Toll-like receptor3; TNBS, trinitrobenzene sulfonic acid.
} 
a
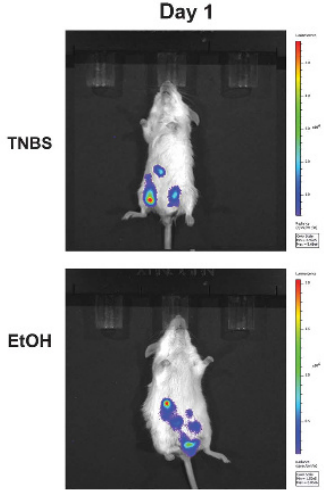

Day 2
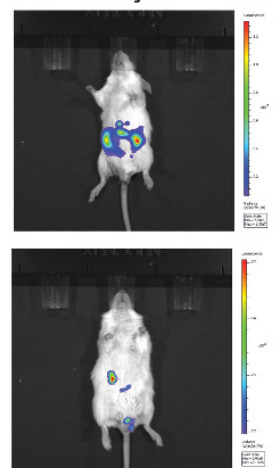

Day 5
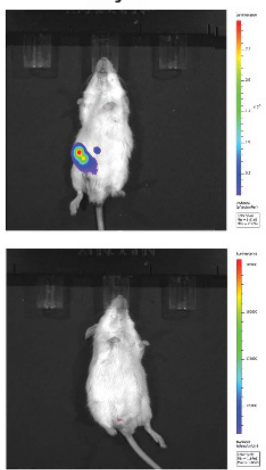

b

Day 4

Intestine

Spleen

MLN
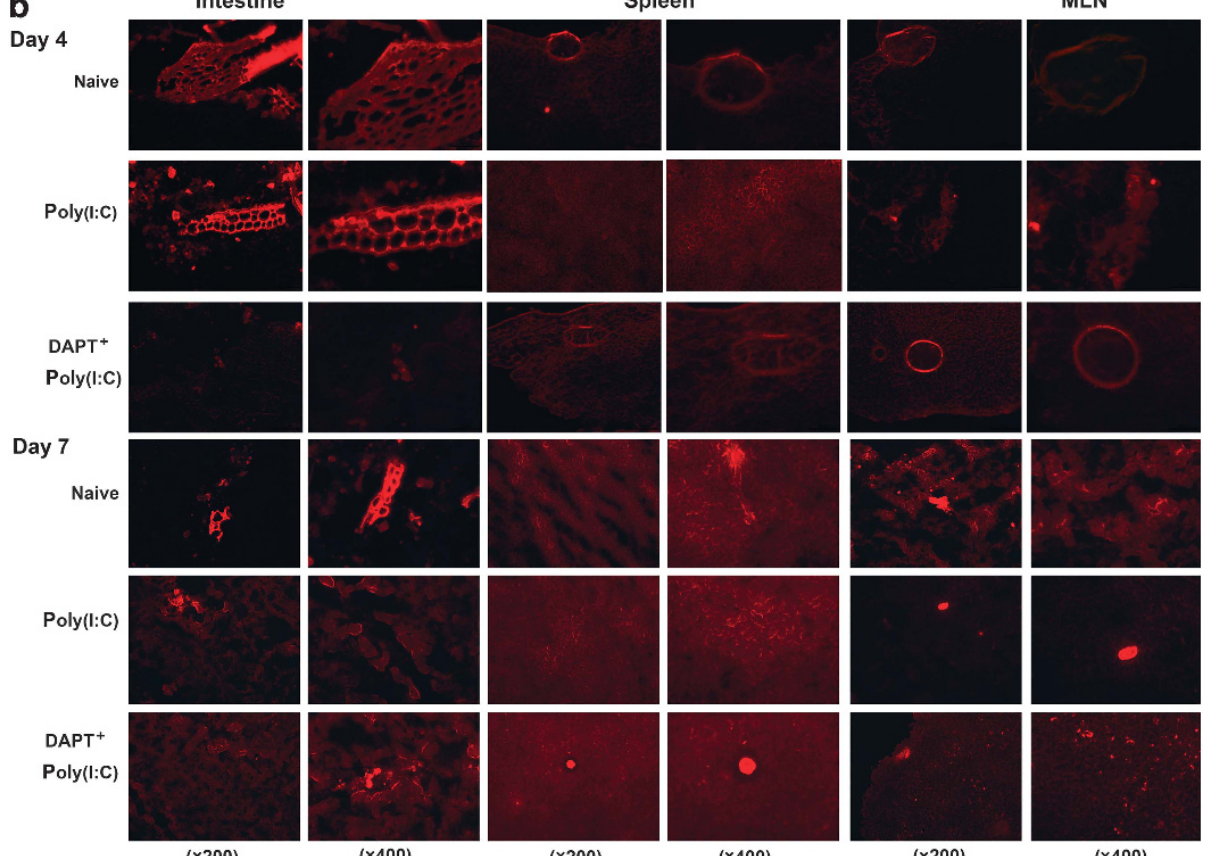

(×200)

$(\times 400)$
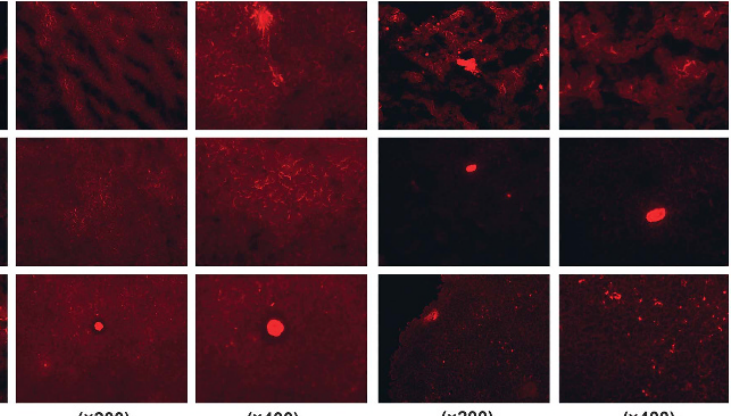

c.
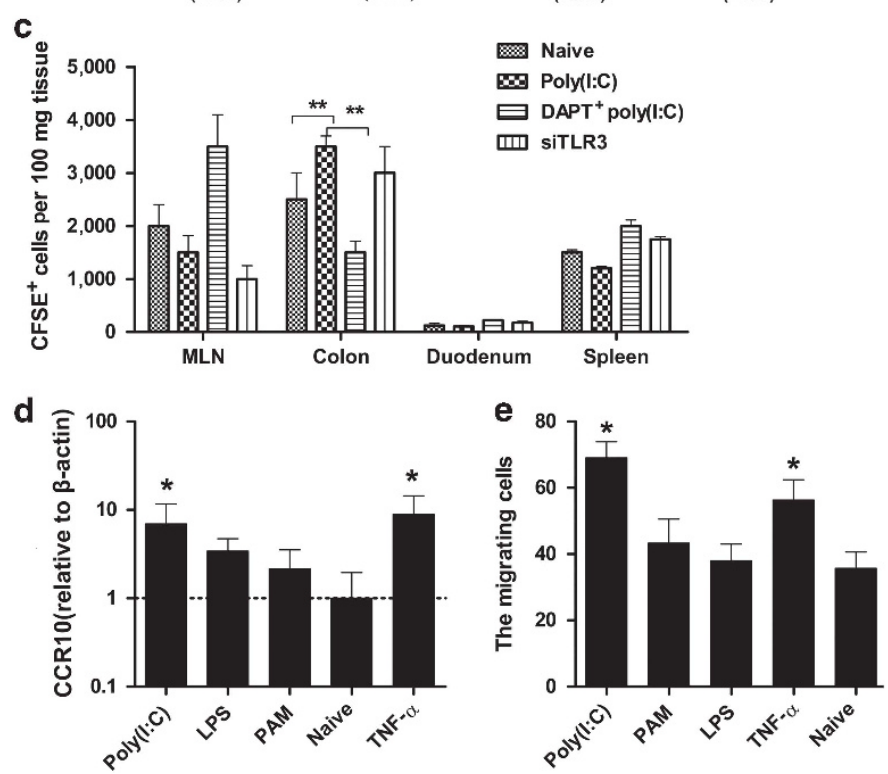

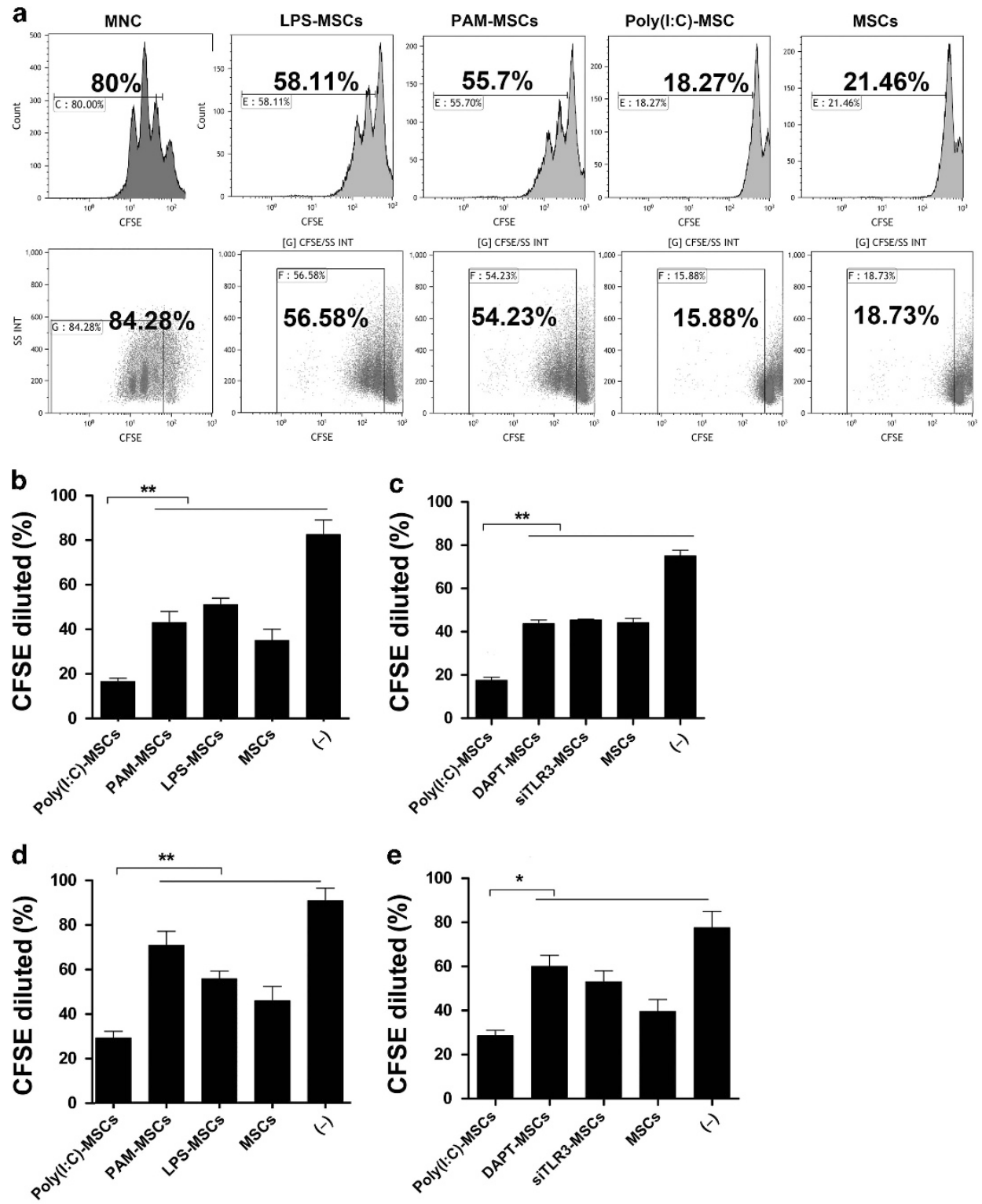

Figure 5 Poly (I:C) enhanced the immunosuppressive effect of hUC-MSCs. (a-c) MNCs $\left(10^{6}\right.$ cells) were stimulated with anti-CD3/CD28 beads and cocultured with hUC-MSCs after treatment with various ligands. MNC proliferation was determined $72 \mathrm{~h}$ later via flow cytometry analysis of the CFSE fluorescence intensity. (d and e) MNCs were cultured with the culture media from hUC-MSCs primed with various ligands. MNC proliferation was determined. The data are presented as the means \pm s.d. of three separate experiments in which five mice per group were analyzed $\left({ }^{\star} P<0.05 ;{ }^{* *} P<0.01\right)$. CFSE, 5-(and-6)-carboxy fluorescein succinimidyl ester; DAPT, NN-(3,5-difluorophenacetyl-L-alanyl)-S-phenylglycine t-butyl ester; hUC-MSC, human umbilical cord-derived MSC; MSC, mesenchymal stem cell; LPS, lipopolysaccharide, a ligand for TLR2; MNC, mononuclear cell; MSC, unstimulated hUC-MSC; poly (I:C)-CM, culture media obtained from hUC-MSCs stimulated with polyribocytidylic acid; siTLR3, small RNA interference targeting TLR3 signaling; TLR3, Toll-like receptor-3; UCM, culture media from unstimulated hUC-MSC.

Figure 4 Poly (I:C) enhanced the migration of hUC-MSCs to inflammatory sites. (a) In vivo bioluminescence imaging of luciferase-expressing fluc-MSC delivery. (b) Ex vivo imaging of CM-Dil dye-labeled MSCs in spleens, MLNs, and colons. Representative fluorescence microscopic colonic slices were obtained on days 4 and 7. (c) Biodistribution of hUC-MSCs. CFSE-labeled hUC-MSCs $\left(10^{6}\right)$ were injected intraperitoneally to TNBS-colitic mice on day 0 , and their presence in various organs was determined via flow cytometry at 4 days after injection. Each bar represents the number of CFSE ${ }^{+}$cells per $100 \mathrm{mg}$ tissue. $n=5$ mice per group. (d) The preferential homing of poly (I:C)-MSCs to inflamed tissues was accompanied by increased expression of CCR10. (e) Activation of TLR3 signaling promoted the migration of hUC-MSCs in vitro in transwell plates. The average number of migrating cells per field was assessed by counting at least four high-power random fields per filter. ${ }^{*} P<0.05$, ${ }^{*} P<0.01$. RQ, relative expression; CFSE, 5-(and-6)-carboxy fluorescein succinimidyl ester; DAPT, $N N$-(3,5-difluorophenacetyl-L-alanyl)-S-phenylglycine $t$-butyl ester; hUC-MSC, human umbilical cord-derived MSC; MSC, mesenchymal stem cell; MLN, mesenteric lymph node; poly (I:C)-MSC, polyribocytidylic acid-stimulated hUC-MSC; siTLR3, small RNA interference targeting TLR3 signaling; TLR3, Toll-like receptor-3. 
showed that hUC-MSCs primed by poly (I:C) were not induced to produce transforming growth factor- $\beta 1$ (TGF- $\beta 1$ ) in the absence of exogenous IFN- $\gamma$ (Figure 6a). Similarly, no increased expression of IL-6 (Figure 6b) or IDO (Figure 6c) was observed upon the precondition of poly (I:C). Our study corroborated the TLR3-driven effect of hMSC secretion of $\mathrm{PGE}_{2}$ and, to a lesser degree, IL-4 and IL-10. In contrast, priming with poly (I:C) induced robust production of $\mathrm{PGE}_{2}$ in hUC-MSCs, which was much more pronounced compared with pretreatment with LPS and IFN- $\gamma$ (Figure 6d). The proliferation of mouse MNCs was significantly inhibited by $\mathrm{PGE}_{2}$ in a dose-dependent manner (Figure 6e). Furthermore, the inhibitory effect of poly (I:C)-CM on MNC proliferation was abolished by indomethacin, a cyclooxygenase (COX) inhibitor (Figure 6e).
Notch signaling is involved in the production of $\mathrm{PGE}_{2}$ by poly (I:C)-primed hUC-MSCs

Notch signaling is critical for TLR3/4-induced immune responses. ${ }^{16}$ Therefore, we investigated whether Notch signaling was also required for COX-2 expression and the production of $\mathrm{PGE}_{2}$ by hUC-MSCs in response to poly (I:C). The expression of TLR3 and Jagged-2 in hUC-MSCs was significantly downregulated by siRNA transfection targeting TLR3 or by DAPT, whereas the expression of Jagged-1 was upregulated (Figure 7a,b). TLR3 stimulation of hMSCs resulted in increased Jagged-1 expression and decreased Jagged-2 expression at both mRNA and protein levels (Figure 7c-e). siRNA-induced TLR3 downregulation caused decreased secretion of $\mathrm{PGE}_{2}$ from hUC-MSCs (Figure 7f), which led to abrogation of the immunosuppressive effect of hUC-MSCs on MNC proliferation (Figure 5d).
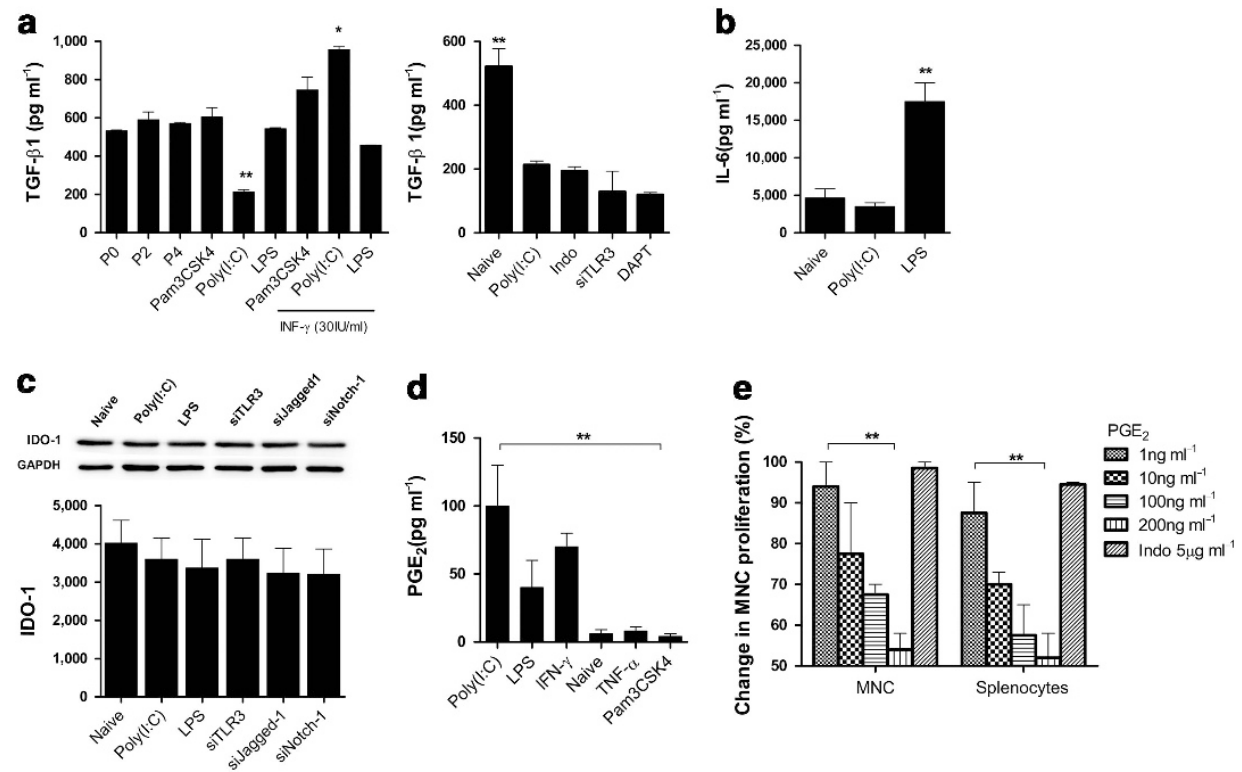

Figure 6 Poly $(\mathrm{I}: \mathrm{C})$-induced $\mathrm{PGE}_{2}$ is responsible for the anti-inflammatory activity of hUC-MSCs. hUC-MSCs were treated with various agonists and the culture media (CM) was collected. (a) TGF- $\beta 1$, (b) IL-6, and (d) PGE 2 concentrations in the CM were determined based on an enzyme-linked immunosorbent assay. (c) Protein levels of IFN- $\gamma$-induced IDO-1 were examined by western blot analysis. (e) MNCs and mouse splenocytes were cultured in the presence of various doses of $\mathrm{PGE}_{2}$, and their proliferation was determined. To further determine whether the effect of $P G E_{2}$ is a key effector of anti-inflammatory activity, indomethacin, a COX inhibitor, was used in the coculture system. ${ }^{*} P<0.05$ and ${ }^{* *} P<0.01$. The data are presented as the means \pm s.d. of three separate experiments in which 5 mice per group were analyzed. hUC-MSC, human umbilical cord-derived MSC; IDO-1, indoleamine 2,3-dioxygenase 1; IFN- $\gamma$, interferon- $\gamma$; IL, interleukin; Indo, indomethacin, a COX inhibitor; LPS, lipopolysaccharide; MNC, mononuclear cell; MSC, unstimulated hUC-MSC; $\mathrm{PGE}_{2}$, prostaglandin $\mathrm{E}_{2}$; poly (I:C), polyribocytidylic acid; TGF- $\beta 1$, tumor growth factor- $\beta 1$.

Figure 7 Poly $(\mathrm{I}: \mathrm{C})$ enhanced the immunosuppressive effect of hUC-MSCs through the TLR3-Jagged-1-Notch-1 pathway. hUC-MSCs were treated with various agonists, and the mRNA expressions of (a) TLR3, (b) Notch-1 and its ligands were determined via reverse transcription-polymerase chain reaction. hUC-MSCs were transfected with siRNAs targeting TLR-3 or Jagged-1 or Notch-1 and then were treated with poly(l:C). The protein levels of TLR3 and Jagged-1/2 were examined via western blot analysis. (c-e and f) Culture media (CM) was harvested from cells treated with poly(l:C) after siRNA transfection. $\mathrm{PGE}_{2}$ secretion was detected from the culture supernatant using an ELISA Kit. (g) MNCs were cultured in the CM, and IL-10 production was measured in the culture supernatant. (h and i) MNCs cultured in CM were analyzed for Th17 and Treg population via flow cytometry. The results correspond to one representative experiment out of three independent experiments. ${ }^{*} P<0.05$ and ${ }^{* *} P<0.01$. The results are shown as the mean \pm s.d. DAPT, $N N$-(3,5-difluorophenacetyl-L-alanyl)-S-phenylglycine $t$-butyl ester; ELISA, enzyme-linked immunosorbent assay; hUC-MSC, human umbilical cord-derived MSC; MNC, mononuclear cell; MSC, unstimulated hUC-MSC; LPS, lipopolysaccharide; PAM, Pam ${ }_{3} \mathrm{CSK}_{4} ; \mathrm{PGE}_{2}$, prostaglandin $\mathrm{E}_{2}$; poly (I:C)-CM, culture media obtained from hUC-MSCs stimulated with poly (I:C); poly (I:C), polyribocytidylic acid; siRNA, small interfering RNA; Th17, T-helper type 17; TLR3, Toll-like receptor-3; UCM, culture media from unstimulated hUC-MSC. 
a
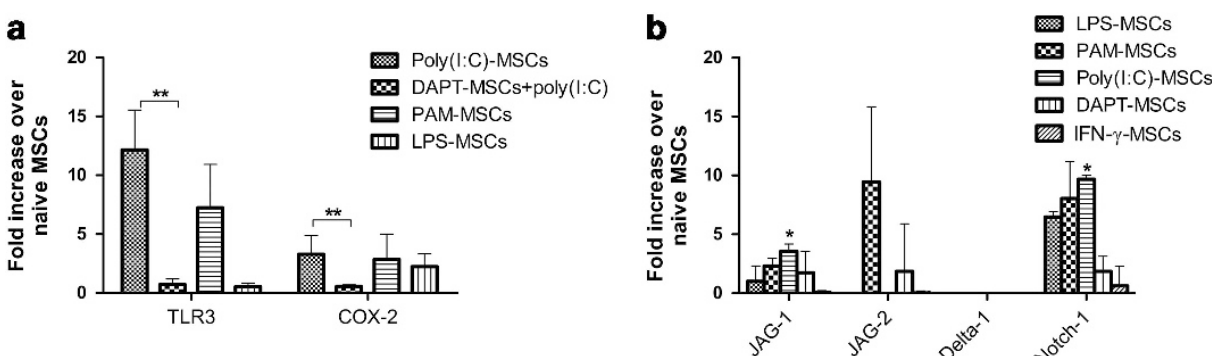

c

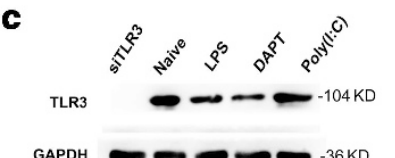

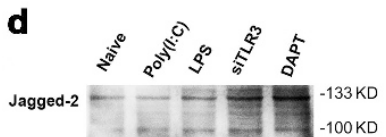

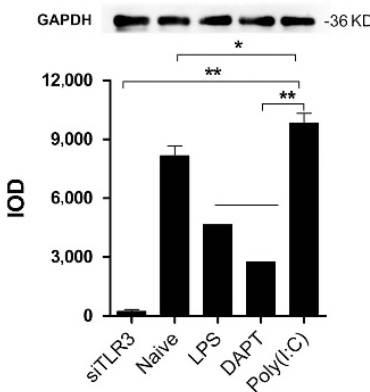

GAPDH - - - $-36 \mathrm{KD}$
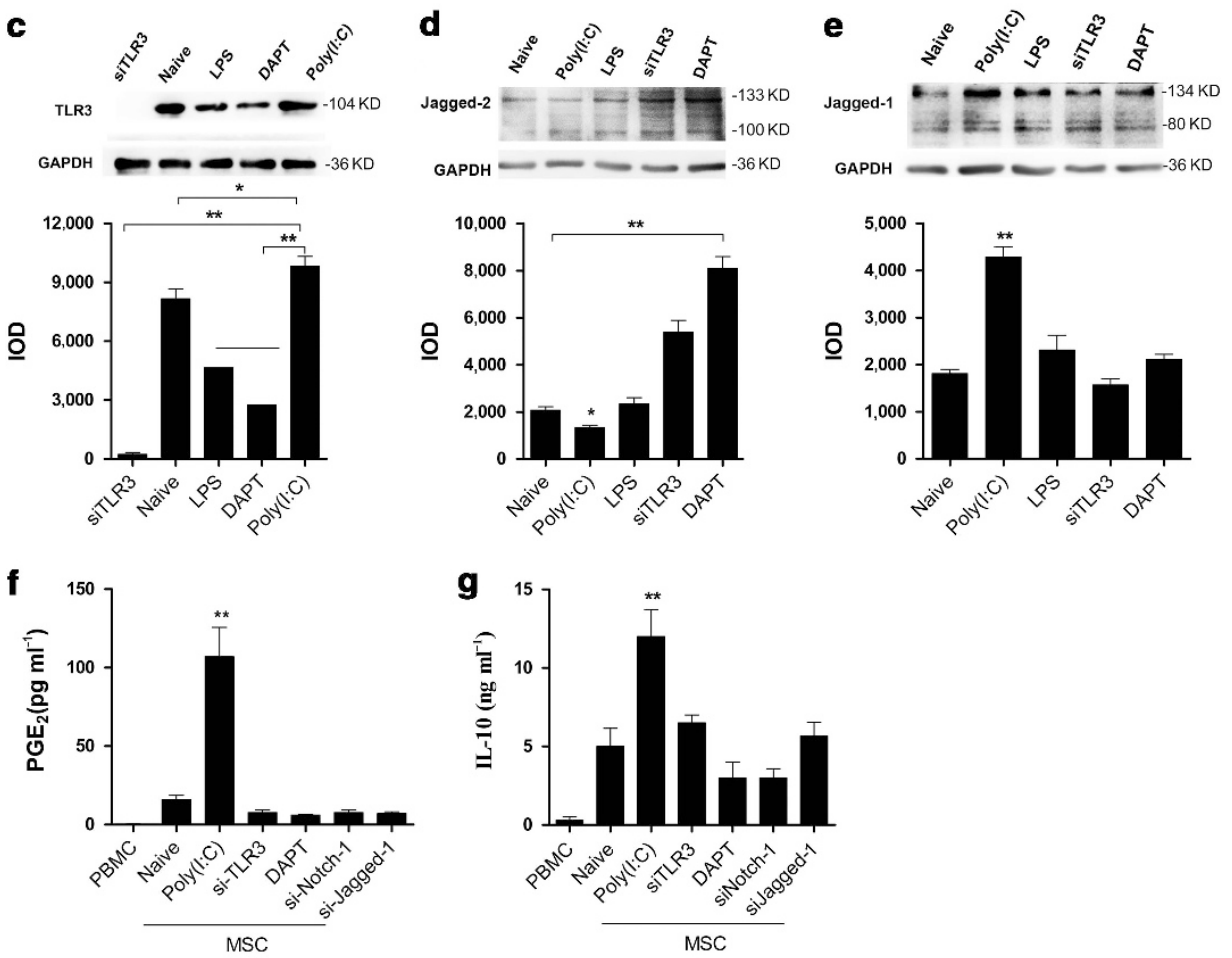

h

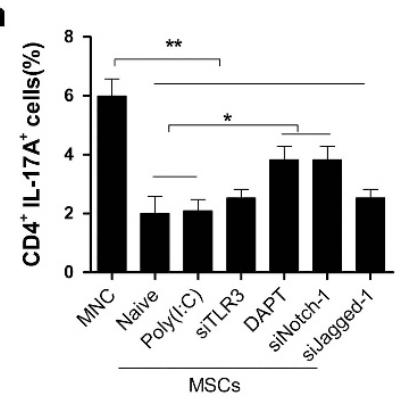

I
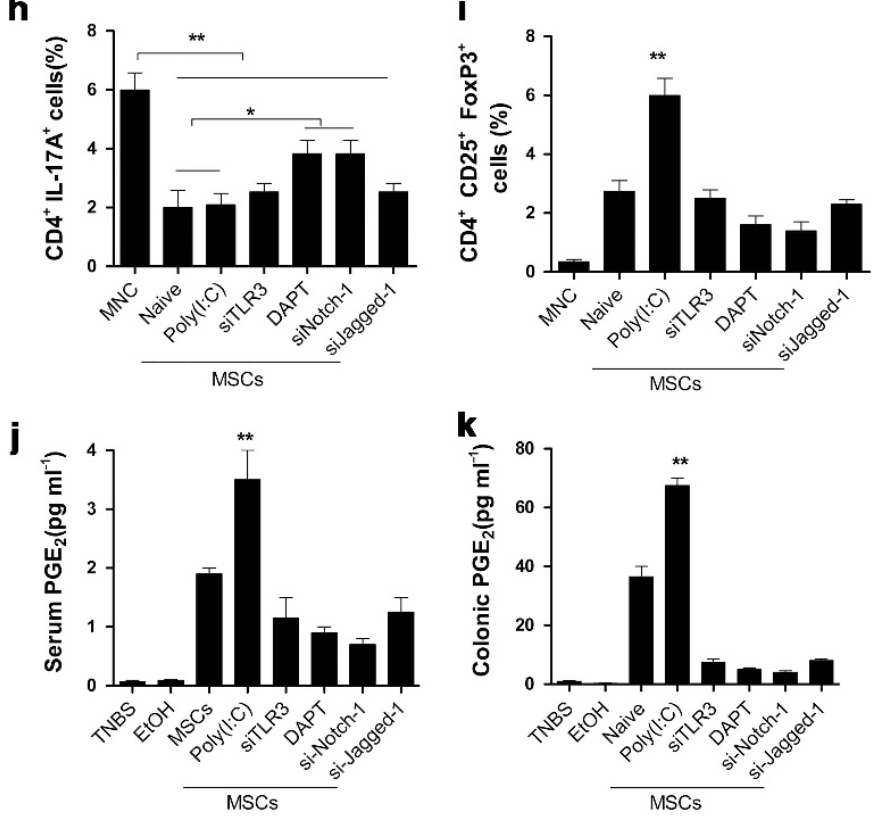


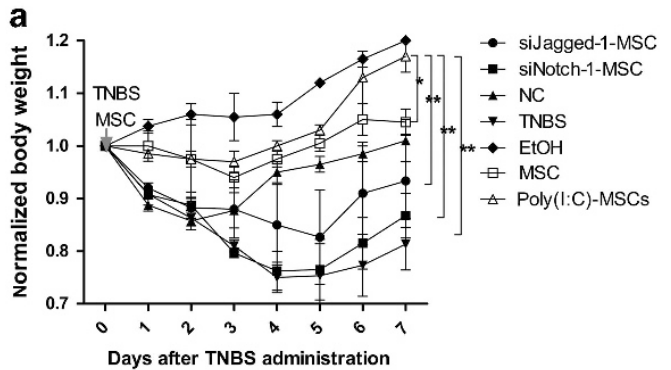

C
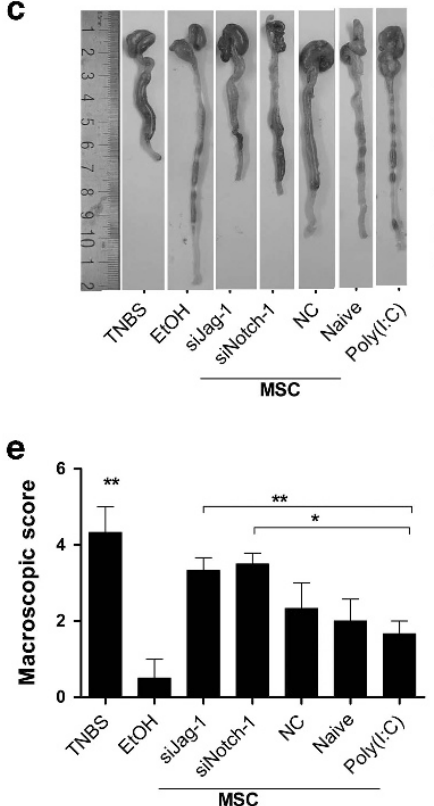

i

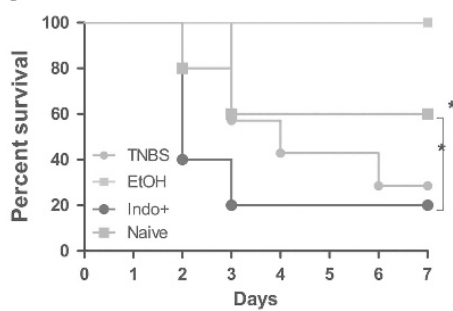

b

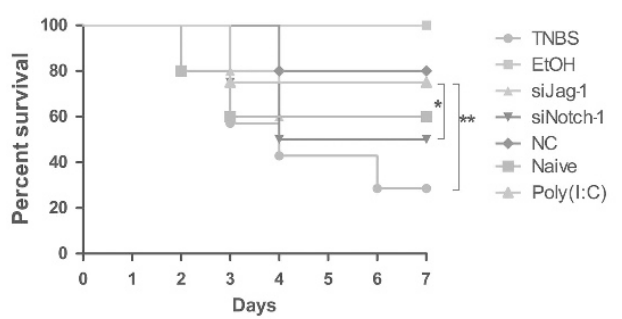

d
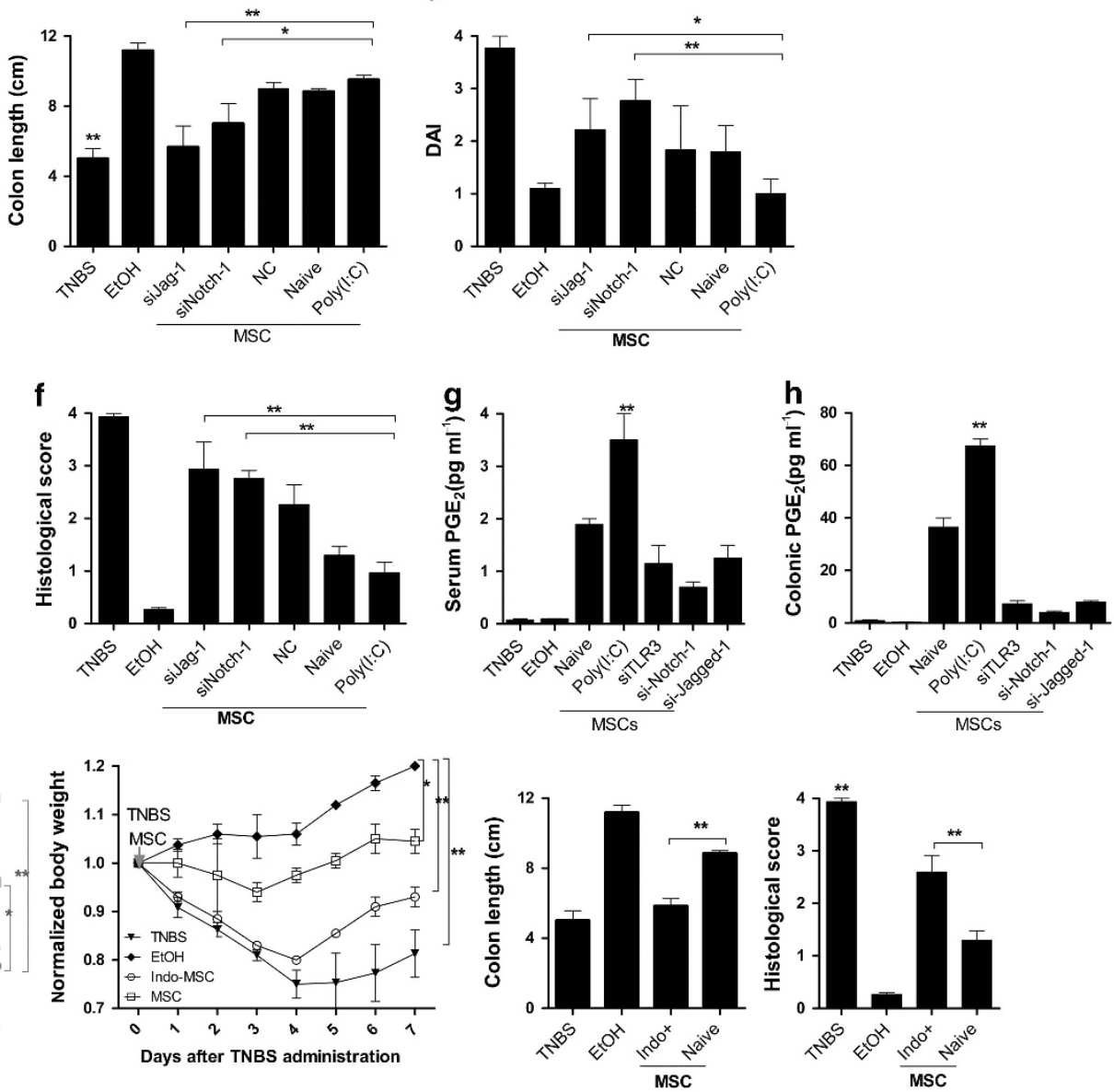

Figure 8 Selective inhibition of the Jagged-1-Notch-1 pathway abolished the immunosuppressive therapeutic effect of hUC-MSCs in colitic mice. siRNA for Jagged-1 or Notch-1 was transfected into hUC-MSCs. Cells were stimulated with poly(I:C) and injected intraperitoneally into TNBS-induced colitic mice. (a) Survival rate, (b) weight loss, (c) colon length, (d) disease activity index and (e and f) histopathologic score were analyzed. ( $\mathbf{g}$ and $\mathbf{h}) \mathrm{PGE}_{2}$ concentration was detected in the TNBS-induced colitic mice. Protein extracts were obtained from the (g) serum and (h) colons on day 4 of hUC-MSC-transplanted colitic mice, and $\mathrm{PGE}_{2}$ was detected using an ELISA Kit. (i) COX-2-inhibited hUC-MSCs were administered into TNBSinduced colitic mice and disease progress was monitored. The results correspond to one representative experiment out of three independent experiments. ${ }^{*} P<0.05,{ }^{*} P<0.01$. The results are shown as the mean \pm s.d. COX, cyclooxygenase; ELISA, enzyme-linked immunosorbent assay; hUC-MSC, human umbilical cord-derived MSC; Indo, indomethacin; NC, group referred to nonsilencing controls; $\mathrm{PGE}_{2}$, prostaglandin $\mathrm{E}_{2}$; TNBS, trinitrobenzene sulfonate.

Although MNCs produced a small amount of IL-10, its production was upregulated in the presence of supernatant from the culture media of UCMs (Figure $7 \mathbf{g}$ ). In addition, siRNAinduced TLR3 or Notch-1 inhibition also inhibited the ability of poly (I:C)-CM to enhance IL-10 production by the MNCs (Figure $7 \mathbf{g}$ ).
We next examined whether UCM affects $\mathrm{CD} 4^{+} \mathrm{T}$ population polarization. The Treg population in the MNCs was increased in the presence of UCM and further increased by poly (I:C)-CM (Figure $7 \mathbf{h}$ ), whereas the Th17 population was decreased (Figure 7i). Similarly, TLR3 or Notch-1 inhibition 
suppressed the ability of poly (I:C)-CM to expand the Treg population (Figure 7i).

\section{Poly (I:C)-mediated robust $\mathrm{PGE}_{2}$ production from hUC-MSCs has a crucial role in the protective effects against colitis in vivo}

To explore the physiological role of $\mathrm{PGE}_{2}$ on the immunosuppressive activity of TLR3-activated hUC-MSCs, Notch-1or Jagged-1-inhibited poly (I:C)-MSCs were administered to colitic mice. Significantly, Notch-1 or Jagged-1 inhibition led to a loss of the ability of poly(I:C)-MSCs to prevent mortality and body weight loss associated with TNBS treatment (Figure 6). Moreover, $\mathrm{PGE}_{2}$ levels in both the serum and the colon of TNBS-induced colitic mice were increased significantly due to hUC-MSC transplantation and further increased by poly (I:C) stimulation but were abolished by the inhibition of TLR3 or Notch-1 (Figure 8g,h).

To explore the physiological role of $\mathrm{PGE}_{2}$ in the immunosuppressive activity of TLR3-activated hUC-MSCs, COX-2inhibited cells were administered to colitic mice. Significantly, COX-2 inhibition abolished the ability of poly(I:C)-MSCs to suppress lethality, body weight loss and disease activity in TNBS-induced colitic mice (Figure 8i).

\section{DISCUSSION}

hUC-MSCs exert immunosuppressive functions, express various TLRs and become activated in response to various inflammatory mediators, ${ }^{11}$ making them an attractive candidate for use in cell therapy strategies of immune-driven inflammatory disorders. In clinical settings, however, the application of hUC-MSCs for the treatment of immune disorders has yielded variable results, possibly because administered MSCs encounter insufficient proinflammatory cytokines or a biased cytokine milieu in vivo. Therefore, when applying MSCs in vivo, it is desirable to drive the polarization of MSCs to the desired immunosuppressive phenotype to optimize their potential clinical use. The main finding of this study was that preconditioning by TLR 3 stimulation selectively enhances the immunosuppressive protective effect of hUCMSCs on experimental colitis. In mice, treatment with poly (I:C)-preconditioned hUC-MSCs abrogated the weight loss, improved the histologic severity and protected against the mortality associated with TNBS-induced colitis, which represents a murine experimental model of Crohn's disease.

The present study further found that poly (I:C)-MSCs rapidly and preferentially migrated to the inflamed colon after intraperitoneal injection, as opposed to their low-magnitude trafficking to other noninflamed gut segments. Chemokines and their receptors are now recognized as important mediators of stem cell homing. To date, the most studied chemokinechemokine receptor axis in MSC homing to wounds is CXCL12-CXCR4, but recent work has suggested that CCL27-CCR10 and CCL21-CCR7 may also be involved. ${ }^{19}$ We found that the preferential homing of poly (I:C)-MSCs to inflamed tissues correlated with the expression of CCR10. In contrast, a relatively smaller fraction of nonprimed MSCs eventually resides in the inflamed colon.

There are several potential mechanisms for the effects of hUC-MSCs on TNBS-induced colitis. Several studies, including our study, have shown that adipose stem cells (ASCs) ${ }^{20}$ and nonprimed hUC-MSCs ${ }^{21}$ downregulate systemic and local inflammatory responses and protect mice against the development of colitis. The upregulation in Th1 activity in TNBSinduced colitis and the reduction in Th1 activity after hUCMSC transplantation were confirmed based on serum and colonic protein levels of INF- $\gamma$ and tumor necrosis factor- $\alpha$ and on the mRNA expression of Stat-4, which is a key component that regulates the expression of Th1 cytokines ${ }^{22}$ (Figure 2f). In addition, there was a further decrease in Th1 activity in acute poly(I:C)-MSC-treated colitis (vs. nonprimed MSC group) (Figure 2). A possible role for hUC-MSCs in promoting the Th2 subset was confirmed by analyzing the protein level of IL-4 via ELISA and the Th2 lineage transcription factor Stat- 6 via real-time PCR (Figure 2f). ${ }^{18}$ Stat-6 expression was further increased in the poly(I:C)-MSCs group. The ROR $\gamma t$ signaling pathway directs the differentiation of proinflammatory Th17 cells. ${ }^{23}$ FACS revealed that poly(I:C)-MSCs clearly inhibited the differentiation of pathogenic Th17 effector cells (Figure 2d,e). In this study, the protein levels of IL-6 and IL-17A and the mRNA expressions of IL-6, IL-17A and ROR $\gamma \mathrm{t}$ were significantly downregulated in the poly(I:C)-MSCs group (Figure 2a-f). The suppressor cytokines IL-10 produced by $\mathrm{CD} 4{ }^{+}$Foxp $3^{+}$Tregs are involved in the control of colitis, as indicated in recent investigations. ${ }^{20}$ hUC-MSCs significantly upregulated IL-10 and Foxp3. The expression of Foxp3 further increased in the poly(I:C)-MSC group compared with the hUCMSC group $(P<0.01$; Figure 2d,e).

Thus far, the specific molecular mechanisms involved remain poorly defined. Previous studies have implied the involvement of both cell-to-cell contact and soluble factors in the deactivation of macrophages and T cells by MSCs. ${ }^{20}$ In this study, stimulation with poly (I:C), but not other agonists, enhanced the inhibitory effect of CM on the proliferation of syngeneic MNCs, which indicates that soluble factors secreted by hUC-MSCs in response to poly (I:C) may have a critical role. It is well known that soluble factors mediate $\mathrm{T}$-cell suppression by MSCs. ${ }^{24}$ Particularly, IDO acts as a switch in human MSCmediated immunomodulation. ${ }^{25}$ In humans, MSC-derived IDO was reported as a requirement for inhibiting the proliferation of IFN- $\gamma$-producing Th1 cells. ${ }^{26}$ Opitz et al. ${ }^{27}$ reported that TLR activation enhances the immunosuppressive activity of bone marrow-derived MSCs (BMSCs) by inducing indoleamine 2,3-dioxygenase 1 (IDO-1), whereas Liotta et al. ${ }^{16}$ demonstrated that TLR3 and TLR4 stimulation do not influence IDO activity. In the present study, no TLR agonists upregulated the expression of IDO-1 in hUC-MSCs. It has been reported that IDO-1 is only released by MSCs after IFN- $\gamma$ stimulation of target cells. ${ }^{25,28,29}$ These findings suggested that, at least in hUC-MSCs, IDO-1 may not be the crucial factor for enhancing hUC-MSC immunosuppressive activity by poly (I:C). 
Moreover, TGF- $\beta,{ }^{30} \mathrm{IL}-6,{ }^{31,32}$ and human leukocyte antigen $\mathrm{G} 5^{33}$ have been reported to be involved in MSC-mediated immune modulation. Mastri et al. ${ }^{13}$ reported that poly(I:C) induced therapeutically relevant trophic factors, such as IL-6type cytokines, in MSCs. Poly(I:C)-treated MSC, but not untreated MSCs, effectively stimulated regeneration of failing hearts in hamsters 1 month after cell administration. TGF- $\beta$ is a multifunctional cytokine that regulates wound healing and tissue repair. ${ }^{34}$ In a previous study, inhibition of TGF- $\beta 1$ signaling abrogated the therapeutic effect of MSC transplantation on dextran sulfate sodium colitis, suggesting a TGF- $\beta 1$ signaling-dependent mechanism. ${ }^{30}$ However, the present study demonstrated decreases in TGF- $\beta 1$ and IL-6, and no increased expression of IDO upon activation of TLR3 when following the protocol by Waterman et al., ${ }^{12}$ as shown in Figure 6a-d, indicating the possible role of other factors. It appeared that TLR4 signaling is upstream of IL-6 and TGF- $\beta$, as demonstrated by Waterman et al. ${ }^{12}$ Our study corroborated the TLR3-driven effect on hMSC secretion of $\mathrm{PGE}_{2}$ and, to a lesser degree, IL-4 and IL-10.

Indeed, we observed that poly (I:C)-MSCs significantly increased $\mathrm{PGE}_{2}$ levels in the supernatants of anti-CD3/CD28stimulated MNCs, suggesting that $\mathrm{PGE}_{2}$ may be the cytokine responsible for the inhibition of lymphocyte proliferation and Treg expansion in MNCs. This hypothesis was further substantiated using indomethacin, a pan COX-2 inhibitor for lymphocyte proliferation. Moreover, transwell separation only slightly reversed the immunomodulatory effect of hUCMSCs and poly (I:C)-MSCs on lymphocyte proliferation, providing evidence that paracrine cytokine and cell contact are the key mechanisms used by poly (I:C)-MSCs.

$\mathrm{PGE}_{2}$ has been reported to mediate most of the immunosuppressive effects that ASCs and BMSCs exert on DC maturation and activated T-cell proliferation. ${ }^{35}$ It acts in synergy with IDO, and MSC-derived $\mathrm{PGE}_{2}$ is able to revert an inflammatory environment to an anti-inflammatory environment, altering the cytokine secretion profile of dendritic and T-cell subsets. ${ }^{36}$ Moreover, a recent study by Chen et al. ${ }^{37}$ showed that $\mathrm{PGE}_{2}$ is critical for the immunosuppressive activity of hUCMSCs, as the inhibition of $\mathrm{PGE}_{2}$ synthesis nearly completely inhibited the immunosuppressive effects of UC-MSCs. Stimulation with poly (I:C), but no other agonists, led to significantly increased expression of COX-2 in hUC-MSCs. ${ }^{38}$ COX-2 is the key molecule in the synthesis and generation of $\mathrm{PGE}_{2}$.

In addition to its direct inhibitory effects on $\mathrm{T}$ cells, $\mathrm{PGE}_{2}$ is known to promote the development of Tregs. ${ }^{39} \mathrm{Kim}$ et al. ${ }^{29}$ previously reported physiological evidence regarding the significance of $\mathrm{PGE}_{2}$ production by transplanted hUC-MSCs and subsequent induction of IL-10-producing Tregs in recipient colitic mice. ${ }^{29}$ Our study also showed that activation of TLR3 enhanced the induction of Treg by hUC-MSCs in a $\mathrm{PGE}_{2}$-dependent manner. IL-10-secreting Treg has a key role in the control of self-antigen-reactive $\mathrm{T}$ cells and the induction of peripheral tolerance in vivo. ${ }^{19}$ To further confirm the critical role of $\mathrm{PGE}_{2}$ in vivo, $\mathrm{COX}$-2-inhibited cells were administered to colitic mice. Significantly, COX-2 inhibition led to a loss of the ability of poly(I:C)-MSCs to prevent mortality and body weight loss associated with TNBS treatment (Figure 8i).

In the current study, we show that Notch-1 signaling is required for hUC-MSCs to exert their immunosuppression roles. Notch signaling regulates immune regulatory effects of both immune cells ${ }^{40}$ and nonimmune cells, such as BMSCs. ${ }^{16,41}$ Using a mouse acute graft-versus-host disease model, Wang et al. ${ }^{41}$ demonstrated that the immunosuppressive function of BMSCs is dependent on Notch signaling and that Notch signaling regulates the immune suppression of BMSCs through the production of IL- 6 and PGE $_{2}$.Disruption of RBP-J, the critical transcription factor that mediates signaling from all four mammalian Notch receptors, remarkably reduced $\mathrm{PGE}_{2}$ production in BMSCs. However, expression of the Notch-1 receptor family member, Jagged-1/2, in TLR-induced MSCs have been linked to controversial reports regarding immunomodulation following TLR activation of MSCs. ${ }^{16,42,43}$ In our study, hUC-MSCs constitutively expressed Jagged-1 but no other Notch ligands, and blocking the Notch transduction pathway with the Notch-1 inhibitor DAPT or siRNA transfection targeting Notch-1 reduced susceptibility to the suppression on T-cell proliferation. Upon implementing the short in vitro TLR-priming protocol reported by Waterman et al., ${ }^{12}$ we found that TLR4-primed hUC-MSCs inhibited the recognized MSC suppression of T-lymphocyte activation, as reported by Liotta et al. ${ }^{16}$ By contrast, Jagged-1 expression was elevated in TLR3-primed hUC-MSCs and, therefore, further activated its signaling to Notch receptors expressed on T cells and enhanced the polarization of T-cell subsets (Supplementary Figure 4 online). ${ }^{44}$ Cahill et al. ${ }^{45}$ recently demonstrated an important role of Jagged-1 in the MSCinduced expansion of Foxp ${ }^{+}$Treg. Furthermore, the ability of poly (I:C)-MSCs to modulate T-cell polarization was abolished by siRNA against Jagged-1, which confirmed a crucial role of Jagged-1. The exact mechanism how $\mathrm{PGE}_{2}$ modulates Notch signaling requires further investigation. $\mathrm{PGE}_{2}$ has been previously reported as a novel regulator of Jagged-1 and a potential microenvironmental modulator of hematopoietic stem cells. ${ }^{46}$

The main finding of this study was that $\mathrm{PGE}_{2}$ shifts the balance from Th1 responses to Th2 responses. The activation of TLR3 enhanced the induction of Tregs by hUC-MSCs in a $\mathrm{PGE}_{2}$-dependent manner. Taken together, these results indicate that TLR3 activation induces $\mathrm{PGE}_{2}$ production by hUCMSCs, which leads to an increase in IL-10 production and Treg population and that the concerted action of $\mathrm{PGE}_{2}$ with subsequent suppressive factors are required for complete attenuation of colitis by hUC-MSCs.

\section{METHODS}

Ethics statement. This study was approved by the Institute Ethics Committee of the First Affiliated Hospital of Sun Yat-sen University and conducted according to the principles expressed in the Declaration of Helsinki. All samples were collected with written informed consent.

MSC culture and expansion. For all sources, MSCs were isolated using the classical adhesion method. When subconfluency (80-90\%) 
was achieved, adherent cells were detached with $0.25 \%$ Trypsin-EDTA (Gibco BRL, Life Technologies, Grand Island, NY) and expanded by replating at a lower density $\left(1,000\right.$ cells per $\left.\mathrm{cm}^{2}\right)$. The MSCs were generally evaluated after two passages. To confirm the mesenchymal nature of the cells, clone-forming unit fibroblast, phenotype, and differentiation assays were performed, as described previously. ${ }^{47}$

MSCs were characterized according to the International Society for Cellular Therapy criteria and as described previously. ${ }^{48}$ Briefly, adherent cells after 3-4 weeks of culture were characterized via flow cytometry using the commercial Human Mesenchymal Stem Cell Marker Antibody Panel according to the manufacturer's instructions. The data were acquired and analyzed using the Kaluza flow cytometry analysis software from Beckman Coulter (Brea, CA).

Multipotency of the hUC-MSCs was tested for differentiation along the osteogenic, and adipogenic lineages using a Mesenchymal Stem Cell Functional Identification Kit (R\&D Systems, Minneapolis, $\mathrm{MN}$ ), as per the manufacturer's instructions. In other experiments, hUC-MSCs were primed with $\mathrm{Pam}_{3} \mathrm{CSK}_{4}\left(1 \mu \mathrm{g} \mathrm{ml}{ }^{-1}\right.$, TLR2), poly (I:C) $\left(1 \mu \mathrm{g} \mathrm{ml}^{-1}\right.$, TLR3), LPS (10 $\mathrm{ng} \mathrm{ml}^{-1}$, TLR4) (SigmaAldrich, St Louis, MO), tumor necrosis factor- $\alpha\left(50 \mathrm{ng} \mathrm{ml}^{-1}\right)$, and/or IFN- $\gamma\left(10^{3} \mathrm{U} \mathrm{ml}^{-1}\right)$ (R\&D Systems, Minneapolis, MN), and subsequently analyzed for cell surface markers via flow cytometry and cytokine secretion using an ELISA. For PGE2 synthesis inhibition experiments, MSCs were resuspended in complete medium in the presence or absence of PGE2 inhibitors indomethacin $(5 \mu \mathrm{M}$; ICN Chemicals, Irvine, CA $)^{12}$ for $48 \mathrm{~h}$, and coculture experiments were then carried out as described in the presence of the inhibitors.

Induction of colitis and study design. The colitic mice model was induced using the classical method, as described previously. ${ }^{20,49}$ Briefly, TNBS ( 3 or $5 \mathrm{mg}$; Sigma) in $50 \%\left(\mathrm{vv}^{-1}\right)$ ethanol $(100 \mu \mathrm{l})$ was administered intrarectally in BALB/c mice. Control mice received $50 \%$ ethanol alone. Animals were treated intraperitoneally with medium or with defined amounts $\left(10^{6}\right.$ cells per mouse) of hUC-MSCs, TLR3activated hUC-MSCs, or syngeneic mASCs (isolated from BALB/c mice) $2 \mathrm{~h}$ after TNBS administration.

Animals were monitored for diarrhea, body weight loss, and survival. At day 4, blood samples and various segments of the colon were collected. Tissue segments were immediately frozen in liquid nitrogen for histologic studies, protein extraction, cytokine determination, and measurement of MPO activity. In vitro models were divided into thirteen groups (Supplementary Table 2).

Intracellular staining. Intracellular staining for IFN- $\gamma$, IL-17, and IL-4 was performed on MSCs after $6 \mathrm{~h}$ of activation with phorbolmyristate acetate $\left(1 \mathrm{ng} \mathrm{ml}^{-1}\right)$ and ionomycin $\left(1 \mu \mathrm{g} \mathrm{ml}^{-1}\right)$ in the presence of Golgi stop brefeldin A ( $10 \mu \mathrm{g} \mathrm{ml}^{-1}$; all from Sigma-Aldrich) for the final $3 \mathrm{~h}$ of culture. The cells were fixed and permeabilized with $\mathrm{BD}$ Cytofix/Cytoperm Plus (BD Bioscience, San Jose, CA) according to the manufacturer's instructions. The cells were incubated with phycoerythrin-Cy7-conjugated rat anti-mouse IFN- $\gamma$, V450-conjugated rat anti-mouse IL-4, and phycoerythrin-conjugated rat anti-mouse IL-17A (all from BD Bioscience). The cells were then washed and data were acquired and evaluated using a Gallios flow cytometer and analyzed using Kaluza Flow Cytometry Analysis Software (Miltenyi Biotec, Bergisch-Gladbach, Germany).

Analysis of Foxp3 expression. MLN cells ( $10^{6}$ cells) were isolated in ice-cold RPMI medium $/ 10 \%$ fetal calf serum and washed two times with phosphate-buffered saline containing $0.1 \%$ sodium azide plus $2 \%$ heat-inactivated fetal calf serum (wash buffer). Cells were incubated with PE/anti-CD4 and APC Cy7/anti-CD25 monoclonal antibodies (BD Pharmingen, San Diego, CA) at $4{ }^{\circ} \mathrm{C}$ for $1 \mathrm{~h}$. After extensive washing, cells were fixed/saponin permeabilized with Cytofix/ Cytoperm solution (BD Biosciences) and incubated for $45 \mathrm{~min}$ at $4{ }^{\circ} \mathrm{C}$ with Alexa-647/anti-Foxp3 monoclonal antibody (BD Biosciences). Then, the cells were washed and analyzed using a Gallios flow cytometer (Brea, CA).
SUPPLEMENTARY MATERIAL is linked to the online version of the paper at http://www.nature.com/mi

\section{DISCLOSURE}

The authors declare no conflict of interest

c) 2017 Society for Mucosal Immunology

\section{REFERENCES}

1. Hanauer, S.B. Inflammatory bowel disease: epidemiology, pathogenesis, and therapeutic opportunities. Inflamm Bowel Dis. 12 (Suppl 1), S3-S9 (2006).

2. Plevy, S.E. \& Targan, S.R. Future therapeutic approaches for inflammatory bowel diseases. Gastroenterology 140, 1838-1846 (2011).

3. Manieri, N.A. \& Stappenbeck, T.S. Mesenchymal stem cell therapy of intestinal disease: are their effects systemic or localized?. Curr. Opin. Gastroenterol. 27, 119-124 (2011).

4. Sudres, $M$ et al. Bone marrow mesenchymal stem cells suppress lymphocyte proliferation in vitro but fail to prevent graft-versus-host disease in mice. J Immunol 176, 7761-7767 (2006).

5. Duijvestein, $M$ et al. Pretreatment with interferon-gamma enhances the therapeutic activity of mesenchymal stromal cells in animal models of colitis. Stem Cells 29, 1549-1558 (2011).

6. Papadopoulou, A. et al. Mesenchymal stem cells are conditionally therapeutic in preclinical models of rheumatoid arthritis. Ann. Rheum. Dis. 71, 1733-1740 (2012).

7. Wu, C.C. et al. TNF-alpha inhibitor reverse the effects of human umbilical cord-derived stem cells on experimental arthritis by increasing immunosuppression. Cell Immunol. 273, 30-40 (2012).

8. Fan, H. et al. Pre-treatment with IL-1beta enhances the efficacy of MSC transplantation in DSS-induced colitis. Cell Mol. Immunol. 9, 473-481 (2012).

9. English, K. Mechanisms of mesenchymal stromal cell immunomodulation. Immunol. Cell Biol. 91, 19-26 (2013).

10. Tomchuck, S.L. et al. Toll-like receptors on human mesenchymal stem cells drive their migration and immunomodulating responses. Stem Cells 26, 99-107 (2008).

11. Chen, D. et al. Expression and role of Toll-like receptors on human umbilical cord mesenchymal stromal cells. Cytotherapy 15, 423-433 (2013).

12. Waterman, R.S. et al. A new mesenchymal stem cell (MSC) paradigm: polarization into a pro-inflammatory MSC1 or an Immunosuppressive MSC2 phenotype. PLoS One 5, e10088 (2010).

13. Mastri, M. et al. Activation of Toll-like receptor 3 amplifies mesenchymal stem cell trophic factors and enhances therapeutic potency. Am. J. Physiol. Cell Physiol. 303, C1021-C1033 (2012).

14. Waterman, R.S. et al. Anti-inflammatory mesenchymal stem cells (MSC2) attenuate symptoms of painful diabetic peripheral neuropathy. Stem Cells Transl. Med. 1, 557-565 (2012).

15. Shi, D. et al. Human adipose tissue-derived mesenchymal stem cells facilitate the immunosuppressive effect of cyclosporin A on T lymphocytes through Jagged-1-mediated inhibition of NF-kappaB signaling. Exp. Hematol. 39, 214-224 e1 (2011).

16. Liotta, F. et al. Toll-like receptors 3 and 4 are expressed by human bone marrow-derived mesenchymal stem cells and can inhibit their T-cell modulatory activity by impairing Notch signaling. Stem Cells 26, 279-289 (2008).

17. Xie, J. et al. Notch signaling regulates CXCR4 expression and the migration of mesenchymal stem cells. Cell. Immunol. 281, 68-75 (2013).

18. Kaplan, M.H. et al. Stat6 is required for mediating responses to IL-4 and for development of Th2 cells. Immunity 4, 313-319 (1996).

19. Hocking, A.M. The role of chemokines in mesenchymal stem cell homing to wounds. Adv. Wound Care (New Rochelle) 4, 623-630 (2015).

20. Gonzalez, M.A. et al. Adipose-derived mesenchymal stem cells alleviate experimental colitis by inhibiting inflammatory and autoimmune responses. Gastroenterology 136, 978-989 (2009).

21. Li, L. et al. Human umbilical cord-derived mesenchymal stem cells downregulate inflammatory responses by shifting the Treg/Th17 profile in experimental colitis. Pharmacology 92, 257-264 (2013).

22. Nishikomori, R. et al. Activated STAT4 has an essential role in Th1 differentiation and proliferation that is independent of its role in the 
maintenance of IL-12R beta 2 chain expression and signaling. J. Immunol. 169, 4388-4398 (2002).

23. Ivanov, I.I. et al. The orphan nuclear receptor RORgammat directs the differentiation program of proinflammatory IL-17 + Thelper cells. Cell 126, 1121-1133 (2006).

24. Ren, G. et al. Mesenchymal stem cell-mediated immunosuppression occurs via concerted action of chemokines and nitric oxide. Cell Stem Cell 2, 141-150 (2008).

25. Meisel, R. et al. Human bone marrow stromal cells inhibit allogeneic T-cell responses by indoleamine 2,3-dioxygenase-mediated tryptophan degradation. Blood 103, 4619-4621 (2004).

26. Krampera, M. et al. Role for interferon-gamma in the immunomodulatory activity of human bone marrow mesenchymal stem cells. Stem Cells 24, 386-398 (2006).

27. Opitz, C.A. et al. Toll-like receptor engagement enhances the immunosuppressive properties of human bone marrow-derived mesenchymal stem cells by inducing indoleamine-2,3-dioxygenase- 1 via interferon-beta and protein kinase R. Stem Cells 27, 909-919 (2009).

28. Ryan, J.M. et al. Interferon-gamma does not break, but promotes the immunosuppressive capacity of adult human mesenchymal stem cells. Clin. Exp. Immunol. 149, 353-363 (2007).

29. Aggarwal, S. \& Pittenger, M.F. Human mesenchymal stem cells modulate allogeneic immune cell responses. Blood 105, 1815-1822 (2005).

30. Wang, C. et al. TGF-beta signaling-dependent alleviation of dextran sulfate sodium-induced colitis by mesenchymal stem cell transplantation. Mol. Biol. Rep. 41, 4977-4983 (2014).

31. Djouad, F. et al. Mesenchymal stem cells inhibit the differentiation of dendritic cells through an interleukin-6-dependent mechanism. Stem Cells 25, 2025-2032 (2007).

32. Jiang, X.X. et al. Human mesenchymal stem cells inhibit differentiation and function of monocyte-derived dendritic cells. Blood 105, 4120-4126 (2005).

33. Nasef, A. et al. Immunosuppressive effects of mesenchymal stem cells: involvement of HLA-G. Transplantation 84, 231-237 (2007).

34. Li, M. O. \& Flavell, R.A. TGF-beta: a master of all Tcell trades. Cell 134, 392404 (2008).

35. Yanez, R. et al. Prostaglandin E2 plays a key role in the immunosuppressive properties of adipose and bone marrow tissue-derived mesenchymal stromal cells. Exp. Cell Res. 316, 3109-3123 (2010).
36. Nauta, A.J. et al. Mesenchymal stem cells inhibit generation and function of both $\mathrm{CD} 34^{+}$-derived and monocyte-derived dendritic cells. J. Immunol. 177, 2080-2087 (2006).

37. Chen, K. et al. Human umbilical cord mesenchymal stem cells hUC-MSCs exert immunosuppressive activities through a PGE2-dependent mechanism. Clin. Immunol. 135, 448-458 (2010).

38. Zhao, X. et al. The toll-like receptor 3 ligand, poly(l:C), improves immunosuppressive function and therapeutic effect of mesenchymal stem cells on sepsis via inhibiting MiR-143. Stem Cells 32, 521-533 (2014).

39. Baratelli, F. et al. Prostaglandin E2 induces FOXP3 gene expression and T regulatory cell function in human CD4 + T cells. J. Immunol. 175, 14831490 (2005).

40. Eagar, T.N. et al. Notch 1 signaling regulates peripheral T cell activation. Immunity 20, 407-415 (2004).

41. Wang, Y.C. et al. Notch-RBP-J signaling is required by bone marrow stromal cells for the treatment of acute graft versus host disease. Stem Cell Res. 11, 721-735 (2013).

42. Samon, J.B. et al. Notch1 and TGFbeta1 cooperatively regulate Foxp3 expression and the maintenance of peripheral regulatory Tcells. Blood 112, 1813-1821 (2008).

43. Blokzijl, A. et al. Cross-talk between the Notch and TGF-beta signaling pathways mediated by interaction of the Notch intracellular domain with Smad3. J. Cell Biol. 163, 723-728 (2003).

44. Amsen, D. et al. Instruction of distinct CD4 T helper cell fates by different notch ligands on antigen-presenting cells. Cell 117, 515-526 (2004).

45. Cahill, E.F. et al. Jagged-1 is required for the expansion of CD4 ${ }^{+} \mathrm{CD} 25^{+}$ $\mathrm{FoxP}^{+}$regulatory $\mathrm{T}$ cells and tolerogenic dendritic cells by murine mesenchymal stromal cells. Stem Cell Res. Ther. 6, 19 (2015).

46. Calvi, L.M. et al. Prostaglandin E-2 (PGE2) regulates osteoblastic Jagged1 and expands primitive hematopoietic cells in vivo. Blood 108, 89 (2006).

47. Bianco, P., Robey, P.G. \& Simmons, P.J. Mesenchymal stem cells: revisiting history, concepts, and assays. Cell Stem Cell 2, 313-319 (2008).

48. Dominici, M. et al. Minimal criteria for defining multipotent mesenchymal stromal cells. The International Society for Cellular Therapy position statement. Cytotherapy 8, 315-317 (2006).

49. Wirtz, S. et al. Chemically induced mouse models of intestinal inflammation. Nat. Protoc. 2, 541-546 (2007). 